OPEN ACCESS

Edited by:

Shu Jiang,

The University of Utah, United States

Reviewed by:

Tianfu Xu,

Jilin University, China

Cao Rui,

Chengdu University of Technology,

China

*Correspondence:

Jun Luo

luojun.syky@sinopec.com

Specialty section:

This article was submitted to

Economic Geology,

a section of the journal

Frontiers in Earth Science

Received: 22 March 2021

Accepted: 30 July 2021

Published: 13 August 2021

Citation:

Zheng H, Luo J, Zhang Y, Feng J, Zeng $Y$ and Wang $M$ (2021) Geological

Characteristics and Distribution of

Granite Geothermal Reservoir in

Southeast Coastal Areas in China.

Front. Earth Sci. 9:683696.

doi: 10.3389/feart.2021.683696

\section{Geological Characteristics and Distribution of Granite Geothermal Reservoir in Southeast Coastal Areas in China}

\author{
Herong Zheng, Jun Luo*, Ying Zhang, Jianyun Feng, Yan Zeng and Mingchuan Wang \\ Sinopec Petroleum Exploration and Production Research Institute, Beijing, China
}

The southeast coastal areas in China have distributed lots of granite outcrops of different periods. Previous research has shown that granite geothermal reservoirs are also distributed under sedimentary basins in these areas, such as in Zhangzhou basin. Therefore, granites with fractures buried in deep can be used as a potential deep geothermal reservoir in these areas. In order to study geological conditions of the deep granite reservoir and discuss the genesis of the deep granite geothermal system, rock geochemistry and zircon $\mathrm{U}-\mathrm{Pb}$ chronology from outcrop and parts of the drilling cores of granitic rocks have been analyzed, combined with the anatomy of the deep seismic data and electromagnetic detection data in selected area. Based on the results of geochemistry and zircon $\mathrm{U}-\mathrm{Pb}$ chronology, most granites in this area are of Yanshanian periods. Based on the seismic data, the thickness of the overlying strata on granite in Huangshadong area of Huizhou City is up to $1.5 \mathrm{~km}$. According to the regional geological survey, multi-stage joints are developed in the granite, and most of hot springs rise from intersection of fracture with different directions to the surface. The heat source in the study area mainly comes from the mantle carried up by the deep NNE-trending faults. There are a large number of thermal springs at the intersection of the surface and the NW-trending fault, and the NWtrending fault provides the drainage conditions for the upwelling of underground thermal springs. There is a huge amount of deep granite geothermal resources in the southeast coastal area. The analysis of deep granite geological conditions and genetic models can provide guidance for the evaluation of deep granite geothermal resources and the further optimization of favorable zones in these areas.

Keywords: southeast coastal areas, granite geothermal reservoir, geothermal resources, geochemistry, geochrohology

\section{INTRODUCTION}

Geothermal resources, as a renewable and clean energy, have been widely used globally in recent years, and the utilization has been increasing year by year (Bertani, 2012; Bertani, 2016; Lund and Boyd, 2016). Recently, most of the geothermal resources under exploitation and utilization are hydrothermal geothermal resource, which is widely distributed in southeastern areas of China (Li Dewei and Wang Yanxin, 2015). Deep geothermal resources are considered as an important part of future energy supply due to their huge thermal energy storage and reserves. 


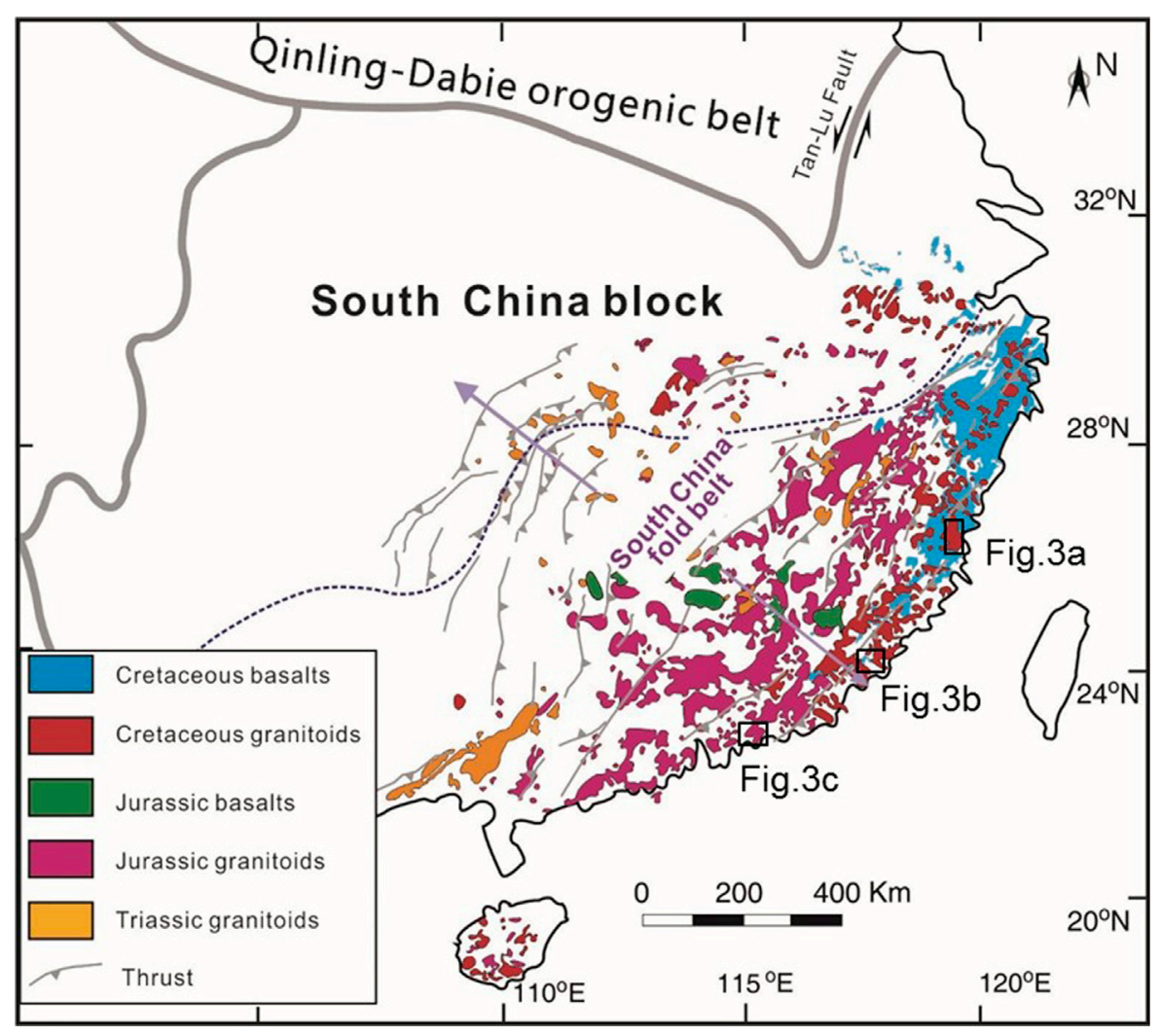

FIGURE 1 | The granite distribution map of the study area (Modified after Zhang et al., 2020).

Most of the deep geothermal reservoir are granite (Brown et al., 2012), and Metamorphic rocks (Li et al., 2019) beneath the sedimentary basins and numerous studies have shown that the granite radioactive heat production has significant contribution to the heat source, such as Australia Cooper basin geothermal field caused by granites with intrusion age less than $0.5 \mathrm{Ma}$ (Goldstein et al., 2008), Rose-manowes geothermal field in England due to Early Permian granite heat generation (Richards et al., 1992), the Soultz geothermal field in France due to Late Paleozoic granite heat generation (Genter et al., 1995).

In the southeast coastal area of China, ground or near surface geothermal manifestations are widely distributed, such as hot springs and other surface heat. They are mainly located in the fractures within igneous rock, such granites. Granites in South China are widely distributed, with an outcropping area of $20,000 \mathrm{~km}^{2}$, accounting for about $1 / 5$ of the area, and it is considered to be formed in three phases, i.e., Caledonian, Indosinian and Yanshanian period (Zhou et al., 2006) (Figure 1). Radioactive elements from those granites such as $\mathrm{U}, \mathrm{Th}, \mathrm{K}$, are important radioactive elements to generate heat by atom decaying. Meanwhile, Southeast coastal areas in China is the second largest region with high heat flow value (Hu et al., 2000). Xi et al. (2018) analyzed the gravity anomaly in Guangdong province and concluded that the decay of thermal elements in granite may be an important part of the geothermal heat sources in the southeast coastal areas. The region is rich in geothermal resources, covering medium-low temperature, medium-high temperature and high-temperature geothermal resources.

In order to further study geological conditions of the deep granite reservoir and discuss the genesis model of the deep granite geothermal system, this paper adopted rock samples from outcrops and parts of the drilling core rocks to analyze the formation background of granite and discuss its geothermal significance to the southeast region in China.

\section{GEOLOGICAL SETTING}

Geologically, the southeast coastal region of China is part of the Cathaysia block (Wang et al., 2013; Deng et al., 2019). These areas have experienced multiple periods of intense magmatic events since the Paleozoic, mainly manifested as a large number of Paleozoic and Mesozoic granitic rocks (Wang et al., 2013) and Cenozoic mafic magmatism (Gong and John, 2014). Large-scale structural deformation caused by emplacements are very strong to the stratum reconstruction, forming a series of faults with different scales and directions.

Granite reservoirs are distributed among the southeastern coastal areas, such as in Zhangzhou, Fuzhou, Fengshun, Yangiiang, and southern Hainan Province. In these areas, the distribution of reservoir is mainly controlled by NE-trending 


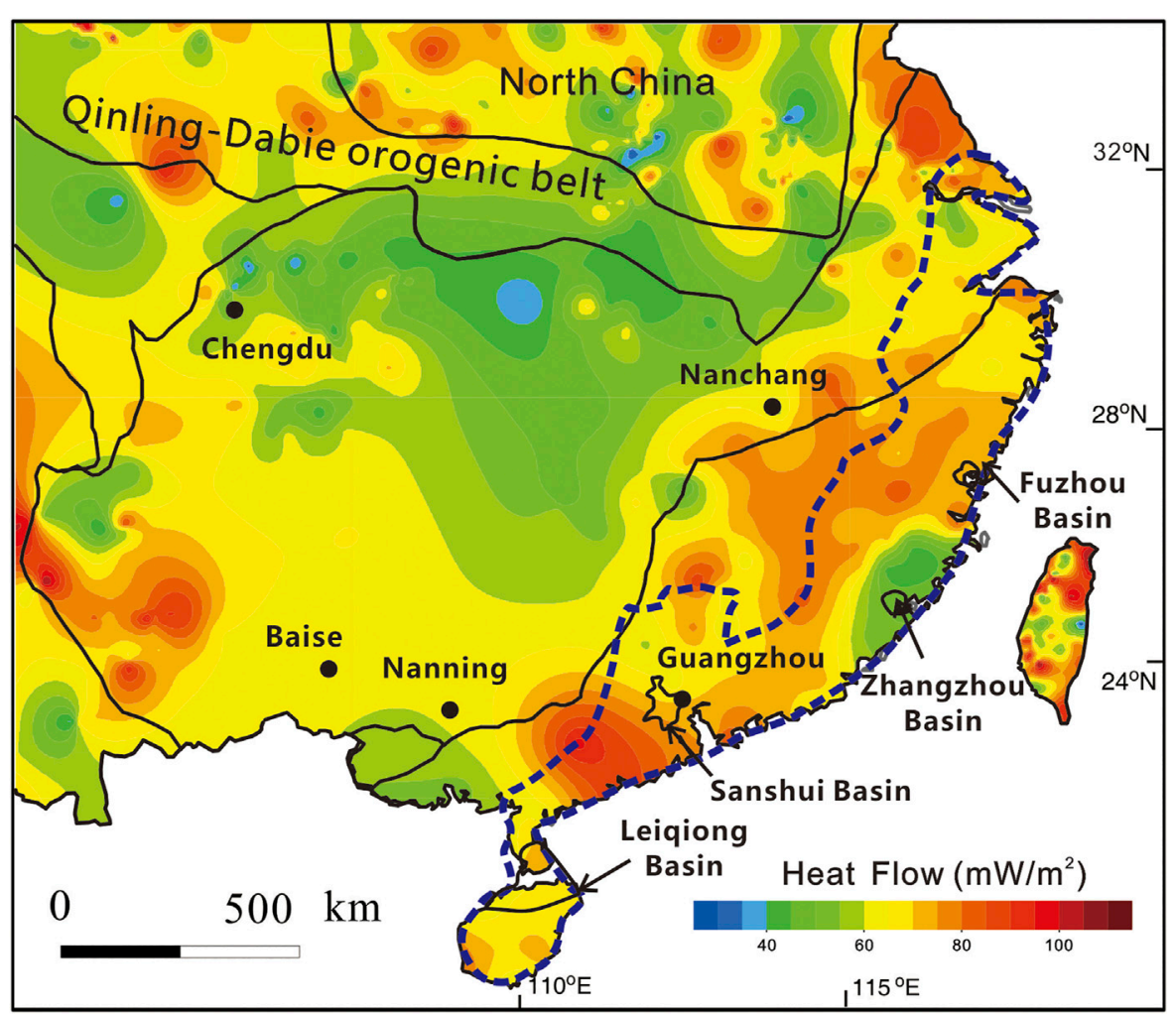

FIGURE 2 | The Heat flow map distribution of the study area (Modified after Zhang et al., 2020).

faults. Most of these granites were formed during the Yanshanian period. There are two types of heat source: Crust source originating from radiogenic heat from $\mathrm{K}$-, Th- and U-bearing minerals of granites and mantle source connecting to the nearby ground by the deep faults. In the Quaternary, most of the radiogenic heat of granite was lost along the fault zone and could not constitute a source of geothermal heat in the fault. Therefore, it is easy to form deep fracture zone and become the channel for hot water to migrate from the deep mantle. In southeast coastal area, $74 \%$ of hot springs are located in fault zone or lithological contact zone in magmatic plutons.

The distribution of most granite geothermal fields are mainly located in basins, such as Fuzhou basin, Zhangzhou basin, Sanshui basin (Figure 1). They are mainly controlled by the deep and large fault in NE direction, and most of them are along the Neo-Cathaysian faults. The zone has experienced many strong tectonic movements and multiple periods of magma intrusion, resulting in secondary faults, rock mass fragmentation and joint fractures near the fault zone, providing space and channels for the storage and migration of geothermal fluids. At the same time, the tectonic activities of deep and large faults not only promote the formation of heat storage space, but also communicate the spatial connection between deep geothermal fluids and shallow geothermal reservoirs, becoming an important heat transfer channel in geothermal fields. From the regional analysis, most of the geothermal fields are linear distribution along the fault zone, mainly exposed on the deep fault axis. The rest are mostly distributed among the deep and large faults, which are locally influenced by the secondary NW tensioned water-conducting faults or pinnate faults. The distribution of heat flow in South China is shown in Figure 2. It contains three obvious thermal structure divisions: Eastern, central and southwestern regions. The eastern and southwestern regions are characterized by high heat flow, while the heat flow in the central region is relatively lower. The heat flow in the eastern and southwestern regions are generally higher than $70 \mathrm{~mW} / \mathrm{m}^{2}$, while that in the central region is lower than $60 \mathrm{~mW} / \mathrm{m}^{2}$. In the eastern region, there are $2 \mathrm{NE}$-trending high value abnormal belts along the Fuzhou-Zhangzhou zone. These two anomaly zones are characterized by high heat flow values $\left(>80 \mathrm{~mW} / \mathrm{m}^{2}\right.$, locally up to $220 \mathrm{~mW} / \mathrm{m}^{2}$ ). The average heat flow in northern Jiangsu Basin is $71.2 \mathrm{~mW} / \mathrm{m}^{2}$, while that in southern Jiangsu and southern Anhui areas is $62.7 \mathrm{~mW} / \mathrm{m}^{2}$. In the central region, from Baise to Dabie Mountains, lower heat flow is distributed along the NE-direction. In the southwestern region, the higher heat flow is concentrated only in southwestern Yunnan. The average of heat flow in the north of Sichuan Basin is $53.6 \mathrm{~mW} / \mathrm{m}^{2}$, while that in southern Yunnan is $76.7 \mathrm{~mW} / \mathrm{m}^{2}$. In the middle of northern Jianghan Basin, the heat flow is lower in the north and higher in the south. The average heat flow in the north of the fault is $47.8 \mathrm{~mW} / \mathrm{m}^{2}$, and that in the south is $61.6 \mathrm{~mW} / \mathrm{m}^{2}$. The average heat flow value of $64.2 \mathrm{~mW} / \mathrm{m}^{2}$ in southern China is higher than that of $61 \mathrm{~mW} / \mathrm{m}^{2}$ in other mainland parts in China (Wang et al., 1990), which is close with that of the globe for $65 \mathrm{~mW} / \mathrm{m}^{2}$ (Yuan, 2006). The 


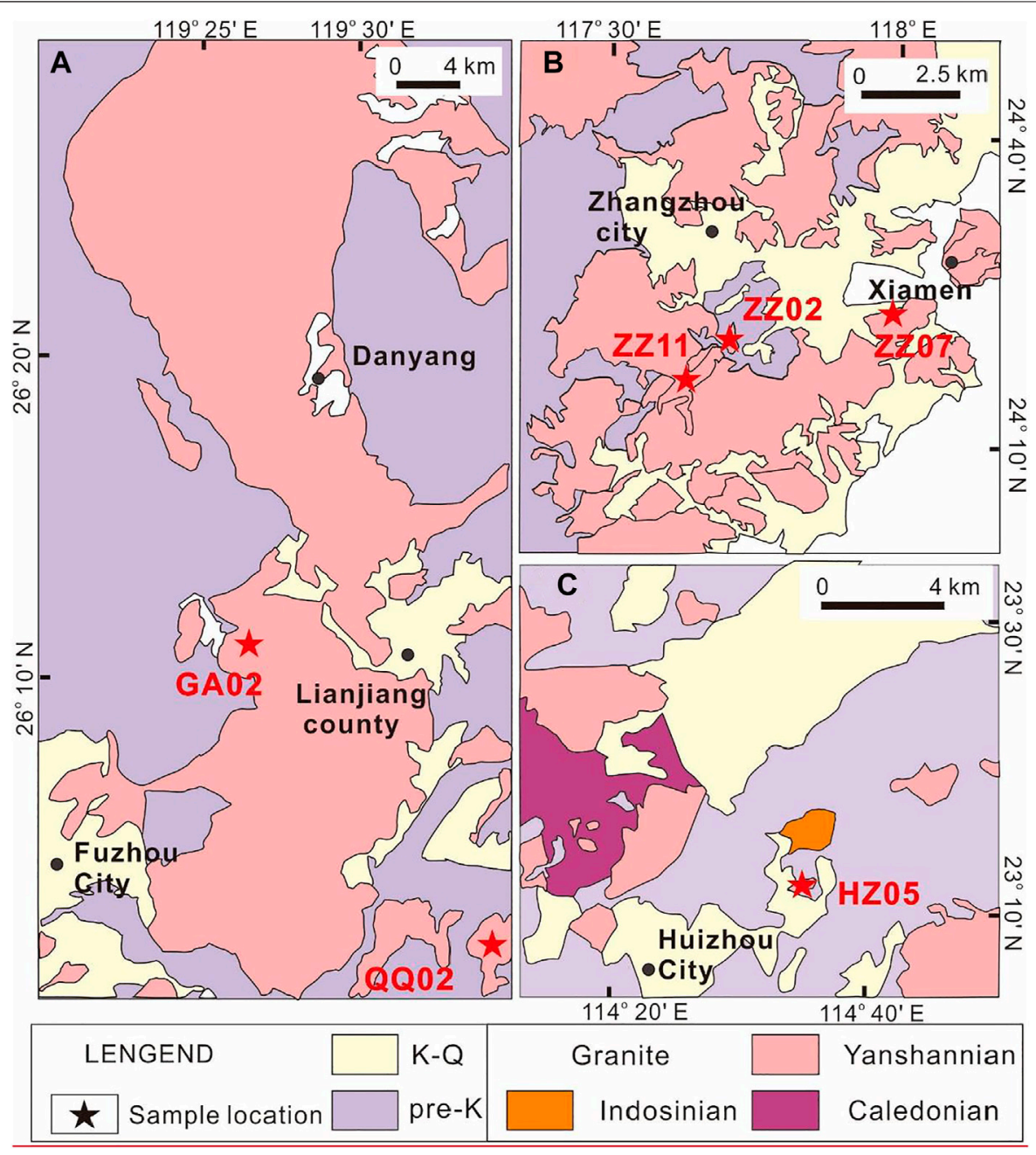

FIGURE 3 | Simplified geological map of selected regions in Southeast Coastal Area: (A) Fuzhou area; (B) Zhangzhou area; (C) Huizhou area (Modified after Zhu 2015; Yang 2016; Zhang et al., 2021).

abnormally high value areas in South China are mainly distributed in the suture zone or regional deep fault zone. Some granite geothermal reservoirs in typical areas in South China have been studied by previous researchers, including Fuzhou, Zhangzhou, Huizhou, Yangjiang areas (Zhu 2015; Yang 2016; Zhang et al., 2021; Zhou, 2015). The Fujian granite geothermal reservoir is located around Fuzhou basin (Figure 3A). Most of them are Yanshanian granites. Early detailed geothermal resource exploration shows that the geothermal reservoir is an area of $9 \mathrm{~km}^{2}$ with a north-south length of about $5 \mathrm{~km}$ and an east-west width of about $2 \mathrm{~km}$. The basement consists of Yanshanian granodiorite, intrusions of medium-coarse biotite granite and fine-grained granite, as well as a large number of intermediate-acid and intermediate-basic dike rocks (Zhu 2015). Zhangzhou granite geothermal reservoir is located around Zhangzhou Basin (Figure 3B). The Quaternary sediments on granite are a set of Marine and continental deposits with a thickness of $20 \mathrm{~m}-30 \mathrm{~m}$. There are several granite rock masses, such as Zhangzhou granite, Chengxi granite and Maping granite, which are around Zhangzhou geothermal field (Yang, 2016). In Huizhou area, Huangshadong geothermal field is located in the northeast of Huizhou city (Figure 3C). This area has experienced several periods of intense magmatic events since the Paleozoic. The granites include Caledonian granodiorite, Indo-Chinese granites, Yanshanian granites and Neogene tholeiitic basaltic dikes (Zhang et al., 2021). Xinzhou complex granite is located in the coastal area of west Guangdong, Yunkai block geologically, on the southwest Cathaysia block, with a total outcropping area of $350 \mathrm{~km}^{2}$. The rock is composed of medium-coarse-grained macro porphyritic biotite monzonite and fine-grained porphyritic biotite quartz syenite, surrounded by the Proterozoic Yunkai Group plagioclase gneiss. The latest outcropped strata around this area are Late Cretaceous sandstone and conglomerate, distributed with NE direction. Xinzhou complex granite mainly includes Xinzhou granite, Dongping granite and Naqin rock granite (Zhou, 2015).

Favorable granite geothermal reservoir is distributed in the exposed magmatic rock mass, near the late intrusive dike or the 

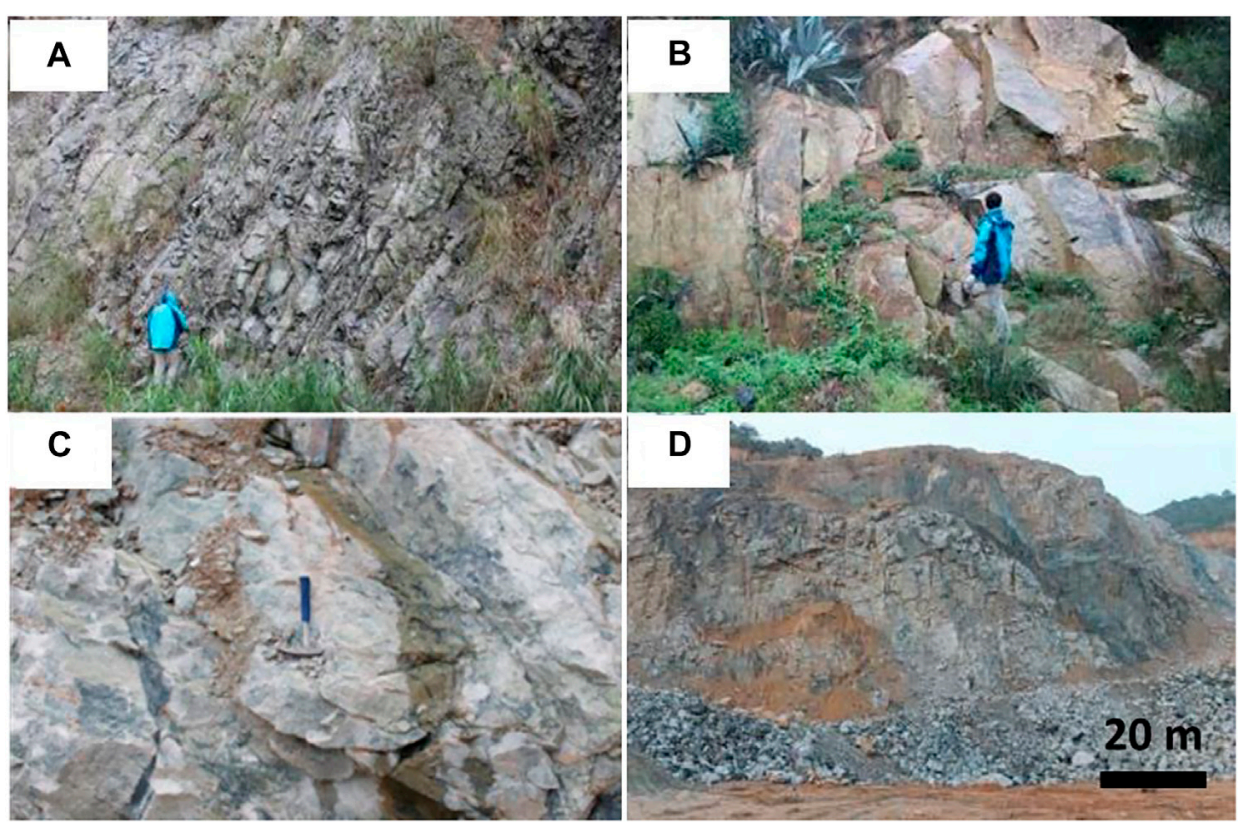

FIGURE 4 |Photograph showing outcrops of granites in different areas, (A) granite outcrop with fractures in Minhou, Fuzhou area; (B) granite outcrops in Qinqian, Fuzhou area; (C) granites with fractures in Huizhou; (D) granites in Zhangzhou.

contact zone between rock mass and surrounding rock mass. The distribution of geothermal field is not only related to the fracture and fracture development of primary rock mass and contact zone rock due to the multi-stage intrusion of magma or the influence of deep fault structure, but also related to the geological age of intrusive rock.

\section{SAMPLE DESCRIPTION AND ANALITICAL METHODS}

In order to interpret magmatism in the study area of granite, a total of 20 samples of magmatic rocks were collected in the selected areas, such as Fuzhou, Zhangzhou and Huizhou area (Figure 3).and they were measured by principal trace element analysis, and zircon U-Pb dating analysis was conducted for six granite samples.

The granite samples collected were mainly distributed along a line of about $1,300 \mathrm{~km}$ with an exposed area of about 10-784 square kilometers. These granites are mainly granitic (Figure 4), with a few being orthogonality, quartz dimorphism and granitic diorite, with granular structure of different thickness and fineness (Figure 5). The mineralogy of these samples is relatively simple, consisting mainly of plagioclase (30-42\%), potash feldspar (30-8\%), quartz (20-30\%), biotite (5\%), and a small amount of amphibole (2-10\%). Accessory minerals include sphere, zircon, and titanium-iron oxides.

$\mathrm{U}-\mathrm{Pb}$ dating and trace element analyses of zircon were conducted synchronously by LA-ICP-MS at the State Key Laboratory of Geological Processes and Mineral Resources, China University of Geosciences, Wuhan. Detailed operating conditions for the laser ablation system and the ICP-MS instrument and data reduction are the same as description by Liu et al. (2010). Laser sampling was performed using a GeoLas 2005. An Agilent 7500a ICP-MS instrument was used to acquire ion-signal intensities. A "wire" signal smoothing device is included in this laser ablation system, by which smooth signals are produced even at very low laser repetition rates down to $1 \mathrm{~Hz}$ (Hu et al., 2012b). Helium was applied as a carrier gas. Argon was used as the make-up gas and mixed with the carrier gas via a T-connector before entering the ICP. Nitrogen was added into the central gas flow $(\mathrm{Ar}+\mathrm{He})$ of the $\mathrm{Ar}$ plasma to decrease the detection limit and improve precision $(\mathrm{Hu}$ et al., 2008a; Liu et al., 2010). Each analysis incorporated a background acquisition of approximately 20-30 s (gas blank) followed by $50 \mathrm{~s}$ of data acquisition from the sample. The Agilent Chemstation was utilized for the acquisition of each individual analysis. Off-line selection and integration of background and analyze signals, and time-drift correction and quantitative calibration for trace element analyses and $\mathrm{U}-\mathrm{Pb}$ dating were performed by ICPMS DataCal (Liu et al., 2008).

Zircon 91,500 was used as external standard for $\mathrm{U}-\mathrm{Pb}$ dating, and was analyzed twice every five analyses. Time-dependent drifts of $\mathrm{U}-\mathrm{Th}-\mathrm{Pb}$ isotopic ratios were corrected using a linear interpolation (with time) for every five analyses according to the variations of 91,500 (i.e., two zircon 91,500 + 5 samples +2 zircon 91,500) (Liu et al., 2010). Preferred U-Th-Pb isotopic ratios used for 91,500 are from Wiedenbeck et al. (1995). Uncertainty of preferred values for the external standard 91,500 was propagated to the ultimate results of the samples. Concordia diagrams and weighted mean calculations were made using Isoplot/Exver3 (Ludwig, 2003). Trace element compositions 


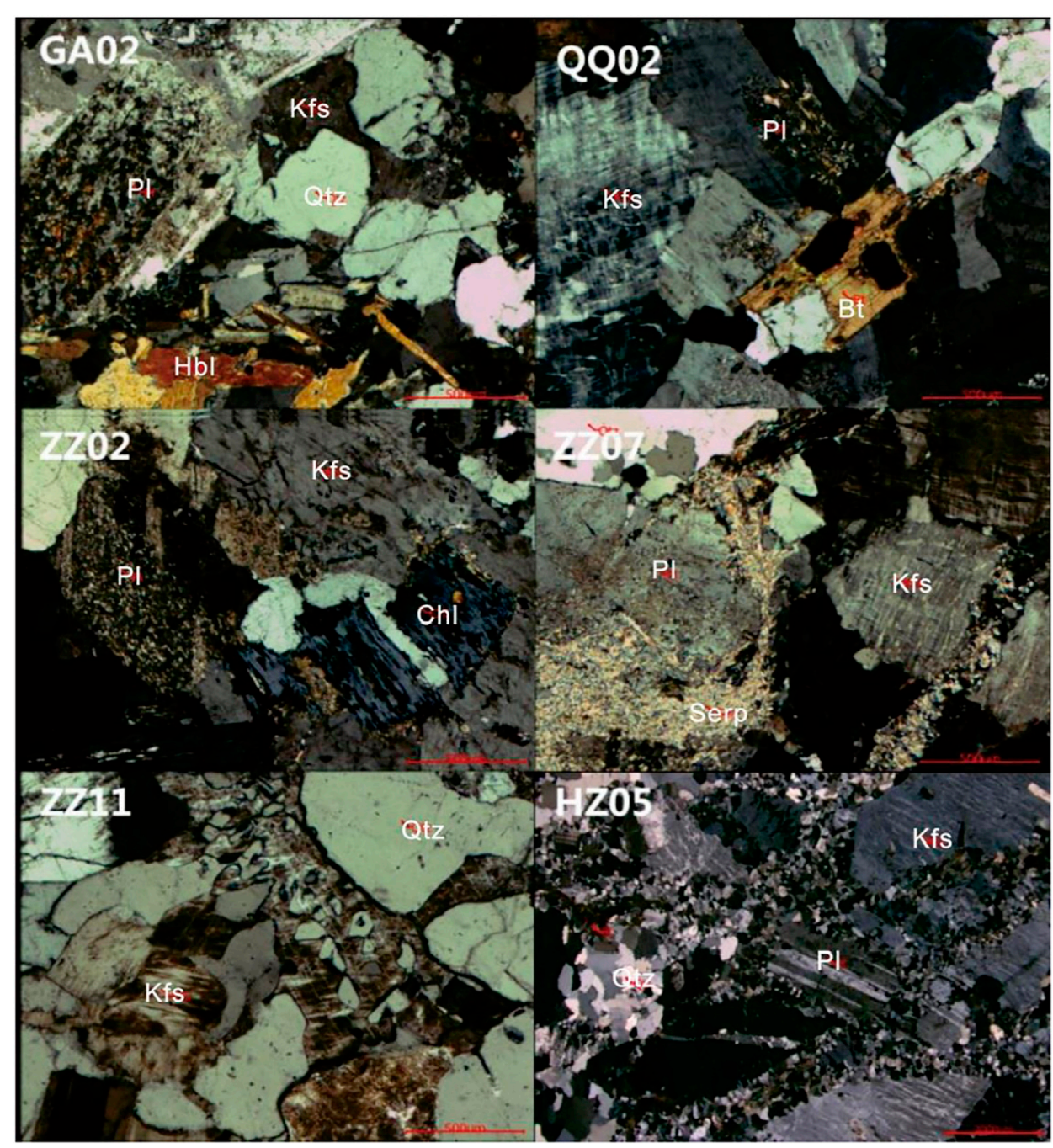

FIGURE 5 | Microphotographs showing mineral assemblage of representative granites. The abbreviations are: PI-plagioclase, Qz-quartz, Bt-biotite, Kfs-Kfeldspar, Amp-amphibole.

of zircons were calibrated against multiple-reference materials (BCR-2G and BIR-1G) combined with internal standardization (Liu et al., 2010). The preferred values of element concentrations for the USGS reference glasses are from the GeoReM database.

\section{RESULTS}

\section{U-Pb Dating}

The concordance ages and analysis results of typical zircons of six samples are shown in Figure 5. They are collected from different areas, such as Fuzhou area (GA02, QQ02) (Figure 3A), Zhangzhou area (ZZ02, ZZ07, ZZ11) (Figure 3B) and Huizhou area (HZ05) (Figure 3C). It can be seen from the CL image that the zircons generally have good autogenesis (Figure 6). Most of them are columnar or long-column-cone shaped with a length of 50-360 m. The aspect ratio of most of them is about 1.5:1-3:1, which is similar to the characteristics of magmatic zircons (Wu Yuanbao and Zheng Yongfei, 2004). The dating results of six samples show that the rock mass in the study area has a wide age distribution, ranging from 94.7 to $138.4 \mathrm{Ma}$. The LA-ICP-MS U-Pb zircon analysis spots are presented in Table 1, and the results are illustrated in Figure 6.

A total of 26 valid data were obtained from GA02. These zircon grains have moderate and variable concentrations of Th (102-200 ppm) and $\mathrm{U}$ (125-318 ppm), corresponding $\mathrm{Th} / \mathrm{U}$ ratios of $0.8-1.14$, showing the characteristic of magmatic zircon (Hoskin and Black, 2000; Sun and McDonough, 1989). These data yield a weighted mean ${ }^{206} \mathrm{~Pb} /{ }^{238} \mathrm{U}$ age of $117.95 \pm$ $0.47 \mathrm{Ma}(\mathrm{MSWD}=0.45)$, representing the formation age of the granite. A total of 28 valid data were obtained from QQ02. These zircon grains have moderate and variable concentrations of Th (127-291 ppm) and U (287-606 ppm), corresponding Th/U ratios of $0.37-0.85$, showing the characteristic of magmatic zircon. These data yield a weighted mean ${ }^{206} \mathrm{~Pb} /{ }^{238} \mathrm{U}$ age of $112.92 \pm 0.85 \mathrm{Ma}(\mathrm{MSWD}=1.6)$, representing the formation 

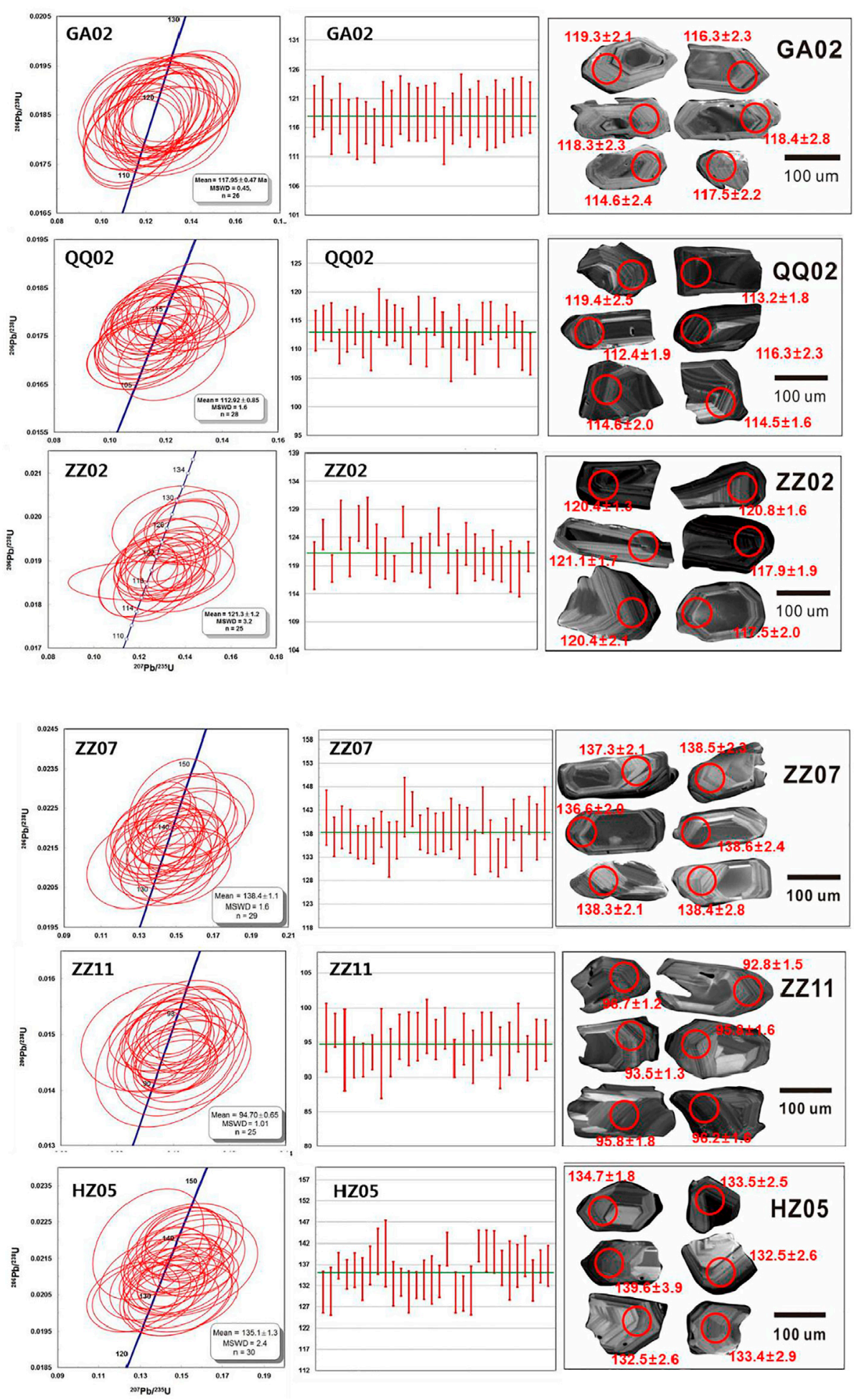

FIGURE 6 | The ${ }^{206} \mathrm{~Pb} /{ }^{238} \mathrm{U}-{ }^{207} \mathrm{~Pb} /{ }^{235} \mathrm{U}$ concordia plot and the weighted mean age of representative zircons of the granites.

age of the granite. A total of 25 valid data were obtained from ZZ02. These zircon grains have moderate and variable concentrations of Th (108-535 ppm) and $\mathrm{U}(154-1,090 \mathrm{ppm})$, corresponding $\mathrm{Th} / \mathrm{U}$ ratios of $0.3-0.99$, showing the characteristic of magmatic zircon. These data yield a weighted mean ${ }^{206} \mathrm{~Pb} /{ }^{238} \mathrm{U}$ age of $121.3 \pm 1.2 \mathrm{Ma}(\mathrm{MSWD}=3.2$ ), representing the formation age of the granite. A total of 29 valid data were obtained from ZZ07. These zircon grains have moderate and variable 
TABLE 1 | Zircon U-Pb data of granites in the study area.

\begin{tabular}{|c|c|c|c|c|c|c|c|c|c|c|c|c|c|c|c|}
\hline \multirow[t]{2}{*}{ Spot } & \multicolumn{3}{|c|}{ Element content } & \multicolumn{6}{|c|}{ Isotope ratio } & \multicolumn{6}{|c|}{ Age (Ma) } \\
\hline & ${ }^{232} \mathrm{Th}$ & ${ }^{238} \mathrm{U}$ & $\begin{array}{c}\text { Th/ } \\
\mathbf{U}\end{array}$ & $\begin{array}{l}{ }^{207} \mathrm{~Pb} / \\
{ }^{206} \mathrm{~Pb}\end{array}$ & 1sigma & $\begin{array}{c}{ }^{207} \mathrm{~Pb} / \\
{ }^{235} \mathrm{U}\end{array}$ & 1sigma & $\begin{array}{c}{ }^{206} \mathrm{~Pb} / \\
{ }^{238} \mathrm{U}\end{array}$ & 1sigma & $\begin{array}{l}{ }^{207} \mathrm{~Pb} / \\
{ }^{206} \mathrm{~Pb}\end{array}$ & 1sigma & $\begin{array}{c}{ }^{207} \mathrm{~Pb} / \\
{ }^{235} \mathrm{U}\end{array}$ & 1sigma & $\begin{array}{c}{ }^{206} \mathrm{~Pb} / \\
{ }^{238} \mathrm{U}\end{array}$ & 1sigma \\
\hline GA02-01 & 200 & 175 & 1.14 & 0.0537 & 0.0042 & 0.1365 & 0.0095 & 0.0186 & 0.0004 & 366.72 & 177.76 & 129.95 & 8.46 & 118.81 & 2.23 \\
\hline GA02-02 & 131 & 150 & 0.87 & 0.0528 & 0.0049 & 0.1334 & 0.0106 & 0.0188 & 0.0004 & 320.43 & 212.94 & 127.11 & 9.49 & 120.27 & 2.29 \\
\hline GA02-03 & 114 & 142 & 0.80 & 0.0530 & 0.0047 & 0.1313 & 0.0105 & 0.0182 & 0.0004 & 327.84 & 197.20 & 125.29 & 9.44 & 116.08 & 2.33 \\
\hline GA02-04 & 114 & 134 & 0.85 & 0.0494 & 0.0053 & 0.1269 & 0.0125 & 0.0187 & 0.0003 & 164.90 & 301.81 & 121.30 & 11.23 & 119.25 & 2.17 \\
\hline GA02-07 & 147 & 170 & 0.86 & 0.0521 & 0.0052 & 0.1271 & 0.0110 & 0.0182 & 0.0004 & 300.06 & 223.12 & 121.51 & 9.94 & 116.25 & 2.30 \\
\hline GA02-08 & 112 & 124 & 0.90 & 0.0465 & 0.0045 & 0.1144 & 0.0092 & 0.0181 & 0.0004 & 20.47 & 218.49 & 109.96 & 8.39 & 115.86 & 2.61 \\
\hline GA02-10 & 370 & 318 & 1.16 & 0.0467 & 0.0032 & 0.1182 & 0.0071 & 0.0183 & 0.0003 & 31.58 & 155.54 & 113.40 & 6.45 & 116.81 & 1.81 \\
\hline GA02-11 & 128 & 149 & 0.86 & 0.0476 & 0.0048 & 0.1126 & 0.0083 & 0.0179 & 0.0004 & 76.02 & 225.89 & 108.33 & 7.53 & 114.63 & 2.29 \\
\hline GA02-12 & 125 & 138 & 0.91 & 0.0491 & 0.0039 & 0.1237 & 0.0076 & 0.0185 & 0.0004 & 153.79 & 174.05 & 118.47 & 6.83 & 118.41 & 2.76 \\
\hline GA02-13 & 164 & 183 & 0.90 & 0.0513 & 0.0044 & 0.1271 & 0.0084 & 0.0184 & 0.0004 & 253.77 & 199.98 & 121.49 & 7.59 & 117.54 & 2.44 \\
\hline GA02-14 & 119 & 135 & 0.88 & 0.0515 & 0.0050 & 0.1316 & 0.0115 & 0.0188 & 0.0004 & 261.18 & 225.90 & 125.50 & 10.33 & 119.89 & 2.51 \\
\hline GA02-15 & 121 & 143 & 0.84 & 0.0492 & 0.0043 & 0.1254 & 0.0095 & 0.0186 & 0.0004 & 166.75 & 187.01 & 119.93 & 8.61 & 118.66 & 2.46 \\
\hline GA02-16 & 142 & 157 & 0.90 & 0.0486 & 0.0041 & 0.1228 & 0.0086 & 0.0185 & 0.0004 & 127.87 & 188.86 & 117.58 & 7.80 & 118.29 & 2.31 \\
\hline GA02-17 & 164 & 172 & 0.96 & 0.0520 & 0.0042 & 0.1280 & 0.0082 & 0.0185 & 0.0004 & 283.40 & 185.16 & 122.33 & 7.39 & 118.47 & 2.39 \\
\hline GA02-18 & 106 & 125 & 0.85 & 0.0465 & 0.0048 & 0.1162 & 0.0099 & 0.0186 & 0.0004 & 33.43 & 224.04 & 111.59 & 9.02 & 118.91 & 2.36 \\
\hline GA02-19 & 108 & 128 & 0.85 & 0.0475 & 0.0045 & 0.1144 & 0.0092 & 0.0179 & 0.0004 & 76.02 & 211.08 & 109.99 & 8.35 & 114.62 & 2.44 \\
\hline GA02-20 & 225 & 226 & 0.99 & 0.0480 & 0.0041 & 0.1188 & 0.0088 & 0.0184 & 0.0003 & 98.24 & 201.82 & 114.02 & 8.00 & & 2.18 \\
\hline GA02-22 & 144 & 159 & 0.90 & 0.0545 & 0.0048 & 0.1380 & 0.0099 & 0.0188 & 0.0004 & 394.50 & 202.75 & 131.25 & 8.79 & 119.92 & 2.65 \\
\hline GA02-23 & 219 & 225 & 0.98 & 0.0526 & 0.0040 & 0.1296 & 0.0086 & 0.0184 & 0.0004 & 309.32 & 174.05 & 123.74 & 7.73 & 117.63 & 2.50 \\
\hline GA02-24 & 134 & 148 & 0.90 & 0.0482 & 0.0045 & 0.1223 & 0.0096 & 0.0187 & 0.0004 & 109.35 & 207.38 & 117.15 & 8.70 & 119.21 & 2.42 \\
\hline GA02-25 & 146 & 161 & 0.91 & 0.0467 & 0.0043 & 0.1160 & 0.0094 & 0.0183 & 0.0004 & 35.28 & 207.38 & 111.39 & 8.56 & 116.70 & 2.30 \\
\hline GA02-26 & 118 & 132 & 0.89 & 0.0481 & 0.0056 & 0.1165 & 0.0110 & 0.0185 & 0.0005 & 105.65 & 261.08 & 111.88 & 10.03 & 118.35 & 2.92 \\
\hline GA02-27 & 191 & 204 & 0.94 & 0.0527 & 0.0044 & 0.1299 & 0.0090 & 0.0185 & 0.0004 & 322.28 & 186.09 & 124.02 & 8.13 & 117.97 & 2.29 \\
\hline GA02-28 & 102 & 126 & 0.81 & 0.0532 & 0.0056 & 0.1314 & 0.0121 & 0.0187 & 0.0004 & 344.50 & 243.49 & 125.34 & 10.89 & 119.43 & 2.54 \\
\hline GA02-29 & 139 & 157 & 0.88 & 0.0523 & 0.0042 & 0.1322 & 0.0092 & 0.0187 & 0.0004 & 298.21 & 183.31 & 126.04 & 8.22 & 119.65 & 2.53 \\
\hline GA02-30 & 181 & 182 & 0.99 & 0.0514 & 0.0043 & 0.1288 & 0.0093 & 0.0187 & 0.0003 & 257.47 & 190.72 & 123.04 & 8.40 & 119.45 & 2.21 \\
\hline QQ02-02 & 246 & 439 & 0.56 & 0.0467 & 0.0028 & 0.1122 & 0.0063 & 0.0177 & 0.0003 & 35.28 & 137.03 & 107.98 & 5.77 & 113.18 & 1.76 \\
\hline QQ02-03 & 261 & 552 & 0.47 & 0.0505 & 0.0028 & 0.1249 & 0.0071 & 0.0179 & 0.0002 & 216.74 & 129.61 & 119.54 & 6.39 & 114.57 & 1.51 \\
\hline QQ02-04 & 261 & 501 & 0.52 & 0.0469 & 0.0025 & 0.1146 & 0.0058 & 0.0180 & 0.0003 & 42.69 & 122.21 & 110.18 & 5.31 & 114.70 & 1.68 \\
\hline QQ02-05 & 291 & 495 & 0.59 & 0.0465 & 0.0025 & 0.1099 & 0.0057 & 0.0173 & 0.0002 & 33.43 & 112.95 & 105.87 & 5.23 & 110.46 & 1.46 \\
\hline QQ02-06 & 210 & 388 & 0.54 & 0.0485 & 0.0031 & 0.1186 & 0.0075 & 0.0177 & 0.0003 & 124.16 & 140.72 & 113.76 & 6.81 & 113.13 & 1.84 \\
\hline QQ02-07 & 317 & 530 & 0.60 & 0.0465 & 0.0024 & 0.1137 & 0.0054 & 0.0179 & 0.0003 & 33.43 & 112.95 & 109.37 & 4.96 & 114.10 & 1.65 \\
\hline QQ02-08 & 251 & 433 & 0.58 & 0.0472 & 0.0031 & 0.1124 & 0.0067 & 0.0176 & 0.0003 & 61.21 & 148.13 & 108.20 & 6.10 & 112.40 & 1.90 \\
\hline QQ02-09 & 265 & 532 & 0.50 & 0.0461 & 0.0025 & 0.1094 & 0.0056 & 0.0172 & 0.0003 & 400.05 & 272.19 & 105.40 & 5.15 & 109.75 & 1.68 \\
\hline QQ02-10 & 176 & 469 & 0.38 & 0.0503 & 0.0026 & 0.1256 & 0.0064 & 0.0182 & 0.0003 & 209.33 & 120.35 & 120.11 & 5.75 & 116.29 & 2.11 \\
\hline QQ02-11 & 254 & 386 & 0.66 & 0.0481 & 0.0031 & 0.1166 & 0.0064 & 0.0179 & 0.0003 & 105.65 & 148.13 & 111.97 & 5.82 & 114.61 & 1.97 \\
\hline QQ02-12 & 236 & 542 & 0.44 & 0.0470 & 0.0023 & 0.1154 & 0.0053 & 0.0179 & 0.0002 & 55.65 & 105.55 & 110.92 & 4.80 & 114.54 & 1.58 \\
\hline QQ02-13 & 373 & 516 & 0.72 & 0.0495 & 0.0025 & 0.1206 & 0.0058 & 0.0178 & 0.0003 & 172.31 & 118.50 & 115.62 & 5.23 & 113.68 & 1.71 \\
\hline QQ02-14 & 250 & 530 & 0.47 & 0.0467 & 0.0028 & 0.1106 & 0.0064 & 0.0173 & 0.0003 & 35.28 & 140.73 & 106.50 & 5.88 & 110.62 & 1.59 \\
\hline QQ02-15 & 233 & 506 & 0.46 & 0.0480 & 0.0026 & 0.1189 & 0.0060 & 0.0181 & 0.0003 & 98.24 & 122.20 & 114.06 & 5.41 & 115.87 & 1.65 \\
\hline QQ02-16 & 151 & 310 & 0.49 & 0.0480 & 0.0035 & 0.1139 & 0.0067 & 0.0173 & 0.0003 & 101.94 & 159.24 & 109.52 & 6.12 & 110.31 & 1.65 \\
\hline QQ02-17 & 153 & 363 & 0.42 & 0.0491 & 0.0030 & 0.1244 & 0.0074 & 0.0181 & 0.0003 & 153.79 & 56.48 & 119.04 & 6.72 & 115.67 & 1.63 \\
\hline QQ02-18 & 167 & 455 & 0.37 & 0.0515 & 0.0030 & 0.1258 & 0.0069 & 0.0177 & 0.0002 & 264.88 & 135.17 & 120.35 & 6.23 & 113.39 & 1.53 \\
\hline QQ02-19 & 168 & 297 & 0.57 & 0.0532 & 0.0041 & 0.1220 & 0.0078 & 0.0171 & 0.0004 & 338.95 & 175.90 & 116.92 & 7.06 & 109.09 & 2.33 \\
\hline QQ02-20 & 208 & 294 & 0.71 & 0.0467 & 0.0032 & 0.1158 & 0.0073 & 0.0178 & 0.0003 & 35.28 & 155.54 & 111.29 & 6.61 & 114.03 & 1.87 \\
\hline QQ02-21 & 146 & 367 & 0.40 & 0.0505 & 0.0034 & 0.1206 & 0.0075 & 0.0175 & 0.0003 & 216.74 & 155.54 & 115.62 & 6.82 & 111.66 & 1.73 \\
\hline QQ02-22 & 225 & 289 & 0.78 & 0.0466 & 0.0032 & 0.1098 & 0.0073 & 0.0171 & 0.0003 & 27.88 & 155.54 & 105.82 & 6.72 & 109.31 & 1.81 \\
\hline QQ02-23 & 268 & 419 & 0.64 & 0.0525 & 0.0040 & 0.1311 & 0.0074 & 0.0179 & 0.0003 & 305.62 & 176.83 & 125.11 & 6.63 & 114.43 & 1.85 \\
\hline QQ02-25 & 192 & 395 & 0.49 & 0.0539 & 0.0032 & 0.1332 & 0.0075 & 0.0180 & 0.0003 & 364.87 & 130.54 & 127.01 & 6.73 & 114.97 & 1.65 \\
\hline QQ02-26 & 189 & 368 & 0.51 & 0.0473 & 0.0032 & 0.1120 & 0.0072 & 0.0173 & 0.0003 & 64.91 & 151.83 & 107.76 & 6.54 & 110.54 & 1.78 \\
\hline QQ02-27 & 281 & 574 & 0.49 & 0.0500 & 0.0030 & 0.1238 & 0.0073 & 0.0180 & 0.0002 & 194.53 & 136.09 & 118.54 & 6.61 & 114.89 & 1.43 \\
\hline QQ02-28 & 517 & 606 & 0.85 & 0.0472 & 0.0027 & 0.1149 & 0.0066 & 0.0177 & 0.0002 & 61.21 & 129.62 & 110.42 & 5.98 & 113.30 & 1.58 \\
\hline QQ02-29 & 127 & 287 & 0.44 & 0.0481 & 0.0035 & 0.1133 & 0.0077 & 0.0173 & 0.0003 & 105.65 & 166.64 & 109.01 & 6.98 & 110.62 & 2.11 \\
\hline QQ02-30 & 152 & 351 & 0.43 & 0.0476 & 0.0036 & 0.1115 & 0.0081 & 0.0171 & 0.0003 & 79.72 & 170.35 & 107.34 & 7.41 & 109.16 & 1.78 \\
\hline ZZO2-01 & 108 & 154 & 0.70 & 0.0546 & 0.0050 & 0.1361 & 0.0105 & 0.0186 & 0.0003 & 394.50 & & 129.56 & 9.35 & 118.98 & 2.08 \\
\hline ZZ02-02 & 418 & 846 & 0.49 & 0.0522 & 0.0025 & 0.1402 & 0.0065 & 0.0195 & 0.0002 & 300.06 & 104.62 & 133.21 & 5.80 & 124.45 & 1.34 \\
\hline ZZO2-03 & 429 & 888 & 0.48 & 0.0528 & 0.0021 & 0.1357 & 0.0052 & 0.0186 & 0.0002 & 320.43 & 88.88 & 129.23 & 4.62 & 118.52 & 1.22 \\
\hline ZZO2-04 & 123 & 216 & 0.57 & 0.0527 & 0.0031 & 0.1433 & 0.0083 & 0.0198 & 0.0003 & 316.73 & 139.80 & 135.99 & 7.34 & 126.17 & 2.19 \\
\hline ZZO2-05 & 209 & 336 & 0.62 & 0.0484 & 0.0030 & 0.1257 & 0.0069 & 0.0189 & 0.0003 & 120.46 & 140.72 & 120.19 & 6.20 & 120.55 & 1.72 \\
\hline ZZO2-07 & 206 & 373 & 0.55 & 0.0511 & 0.0027 & 0.1405 & 0.0075 & 0.0198 & 0.0002 & 242.66 & 122.21 & 133.51 & 6.65 & 126.43 & 1.57 \\
\hline
\end{tabular}


TABLE 1 | (Continued) Zircon U-Pb data of granites in the study area.

\begin{tabular}{|c|c|c|c|c|c|c|c|c|c|c|c|c|c|c|c|}
\hline \multirow[t]{2}{*}{ Spot } & \multicolumn{3}{|c|}{ Element content } & \multicolumn{6}{|c|}{ Isotope ratio } & \multicolumn{6}{|c|}{ Age (Ma) } \\
\hline & ${ }^{232} \mathrm{Th}$ & ${ }^{238} \mathrm{U}$ & $\begin{array}{c}\text { Th/ } \\
\mathbf{U}\end{array}$ & $\begin{array}{c}{ }^{207} \mathrm{~Pb} / \\
{ }^{206} \mathrm{~Pb}\end{array}$ & 1sigma & $\begin{array}{c}{ }^{207} \mathrm{~Pb} / \\
{ }^{235} \mathrm{U}\end{array}$ & 1sigma & $\begin{array}{c}{ }^{206} \mathrm{~Pb} / \\
{ }^{238} \mathrm{U}\end{array}$ & 1sigma & $\begin{array}{c}{ }^{207} \mathrm{~Pb} / \\
{ }^{206} \mathrm{~Pb}\end{array}$ & 1sigma & $\begin{array}{c}{ }^{207} \mathrm{~Pb} / \\
{ }^{235} \mathrm{U}\end{array}$ & 1sigma & $\begin{array}{c}{ }^{206} \mathrm{~Pb} / \\
{ }^{238} \mathrm{U}\end{array}$ & 1sigma \\
\hline ZZ02-08 & 204 & 207 & 0.99 & 0.0499 & 0.0042 & 0.1330 & 0.0102 & 0.0198 & 0.0004 & 187.12 & 188.86 & 126.75 & 9.13 & 126.56 & 2.28 \\
\hline ZZ02-09 & 135 & 185 & 0.73 & 0.0507 & 0.0036 & 0.1308 & 0.0079 & 0.0191 & 0.0004 & 233.40 & 164.79 & 124.80 & 7.07 & 121.82 & 2.24 \\
\hline ZZO2-13 & 136 & 247 & 0.55 & 0.0497 & 0.0033 & 0.1266 & 0.0073 & 0.0188 & 0.0003 & 188.97 & 153.68 & 121.08 & 6.61 & 120.23 & 1.79 \\
\hline ZZ02-14 & 535 & 1090 & 0.49 & 0.0474 & 0.0051 & 0.1208 & 0.0131 & 0.0186 & 0.0002 & 77.87 & 231.45 & 115.76 & 11.85 & 118.49 & 1.15 \\
\hline ZZO2-15 & 426 & 933 & 0.46 & 0.0513 & 0.0024 & 0.1406 & 0.0057 & 0.0199 & 0.0002 & 253.77 & 112.02 & 133.57 & 5.03 & 126.74 & 1.36 \\
\hline ZZ02-16 & 512 & 786 & 0.65 & 0.0480 & 0.0020 & 0.1244 & 0.0051 & 0.0189 & 0.0002 & 98.24 & 96.29 & 119.01 & 4.58 & 120.42 & 1.25 \\
\hline ZZO2-17 & 263 & 673 & 0.39 & 0.0520 & 0.0033 & 0.1354 & 0.0061 & 0.0189 & 0.0002 & 287.10 & 116.65 & 128.94 & 5.44 & 120.84 & 1.57 \\
\hline ZZ02-18 & 110 & 163 & 0.68 & 0.0535 & 0.0044 & 0.1321 & 0.0088 & 0.0188 & 0.0004 & 350.06 & 187.01 & 126.02 & 7.93 & 119.89 & 2.37 \\
\hline ZZ02-19 & 148 & 283 & 0.52 & 0.0505 & 0.0033 & 0.1384 & 0.0085 & 0.0197 & 0.0003 & 220.44 & 151.83 & 131.64 & 7.59 & 125.86 & 1.66 \\
\hline ZZ02-20 & 189 & 311 & 0.61 & 0.0492 & 0.0031 & 0.1288 & 0.0079 & 0.0190 & 0.0003 & 166.75 & 146.28 & 123.00 & 7.12 & 121.13 & 1.71 \\
\hline ZZ02-21 & 179 & 218 & 0.82 & 0.0479 & 0.0037 & 0.1213 & 0.0079 & 0.0184 & 0.0003 & 94.54 & 238.86 & 116.27 & 7.13 & 117.85 & 1.91 \\
\hline ZZ02-22 & 176 & 278 & 0.63 & 0.0504 & 0.0032 & 0.1307 & 0.0070 & 0.0192 & 0.0003 & 213.04 & 144.43 & 124.77 & 6.30 & 122.89 & 1.86 \\
\hline ZZ02-23 & 237 & 416 & 0.57 & 0.0534 & 0.0034 & 0.1380 & 0.0071 & 0.0189 & 0.0003 & 342.65 & 142.57 & 131.23 & 6.37 & 120.42 & 2.06 \\
\hline ZZ02-24 & 504 & 1001 & 0.50 & 0.0535 & 0.0017 & 0.1384 & 0.0043 & 0.0188 & 0.0002 & 350.06 & 78.70 & 131.62 & 3.88 & 119.78 & 1.13 \\
\hline ZZ02-26 & 201 & 223 & 0.90 & 0.0527 & 0.0045 & 0.1375 & 0.0096 & 0.0189 & 0.0003 & 316.73 & 196.27 & 130.79 & 8.61 & 120.86 & 2.19 \\
\hline ZZ02-27 & 183 & 604 & 0.30 & 0.0497 & 0.0024 & 0.1271 & 0.0051 & 0.0187 & 0.0002 & 188.97 & 119.43 & 121.52 & 4.58 & 119.21 & 1.54 \\
\hline ZZ02-28 & 125 & 184 & 0.68 & 0.0480 & 0.0036 & 0.1197 & 0.0071 & 0.0186 & 0.0004 & 101.94 & 231.45 & 114.78 & 6.40 & 118.78 & 2.25 \\
\hline ZZ02-29 & 255 & 261 & 0.98 & 0.0509 & 0.0036 & 0.1258 & 0.0070 & 0.0184 & 0.0003 & 235.25 & 164.79 & 120.28 & 6.31 & 117.50 & 2.01 \\
\hline ZZO2-30 & 248 & 720 & 0.34 & 0.0526 & 0.0020 & 0.1372 & 0.0047 & 0.0189 & 0.0002 & 309.32 & 88.88 & 130.57 & 4.24 & 120.59 & 1.30 \\
\hline ZZ07-01 & 74.8 & 156 & 0.48 & 0.0519 & 0.0046 & 0.1521 & 0.0121 & 0.0222 & 0.0005 & 283.40 & 203.68 & 143.79 & 10.62 & 141.40 & 2.91 \\
\hline ZZ07-02 & 165 & 272 & 0.61 & 0.0463 & 0.0032 & 0.1384 & 0.0090 & 0.0215 & 0.0003 & 13.06 & 159.25 & 131.61 & 8.05 & 137.29 & 2.06 \\
\hline ZZ07-03 & 99.4 & 234 & 0.43 & 0.0461 & 0.0035 & 0.1399 & 0.0102 & 0.0219 & 0.0004 & 400.05 & 224.04 & 132.97 & 9.08 & 139.37 & 2.24 \\
\hline ZZ07-04 & 137 & 232 & 0.59 & 0.0463 & 0.0032 & 0.1390 & 0.0092 & 0.0217 & 0.0004 & 13.06 & 159.25 & 132.12 & 8.21 & 138.51 & 2.30 \\
\hline ZZ07-05 & 219 & 355 & 0.62 & 0.0524 & 0.0039 & 0.1530 & 0.0104 & 0.0214 & 0.0003 & 301.91 & 168.50 & 144.54 & 9.18 & 136.21 & 1.76 \\
\hline ZZ07-05 & 219 & 355 & 0.62 & 0.0524 & 0.0039 & 0.1530 & 0.0104 & 0.0214 & 0.0003 & 301.91 & 168.50 & 144.54 & 9.18 & 136.21 & 1.76 \\
\hline ZZ07-06 & 118 & 201 & 0.59 & 0.0526 & 0.0045 & 0.1486 & 0.0102 & 0.0214 & 0.0004 & 322.28 & 196.27 & 140.72 & 9.06 & 136.44 & 2.42 \\
\hline ZZ07-07 & 178 & 361 & 0.49 & 0.0478 & 0.0025 & 0.1445 & 0.0077 & 0.0218 & 0.0003 & 87.13 & 118.50 & 137.07 & 6.80 & 138.94 & 1.88 \\
\hline ZZ07-08 & 50.5 & 93.8 & 0.54 & 0.0480 & 0.0058 & 0.1314 & 0.0119 & 0.0211 & 0.0005 & 98.24 & 262.93 & 125.33 & 10.65 & 134.59 & 2.91 \\
\hline ZZ07-10 & 118 & 324 & 0.36 & 0.0464 & 0.0031 & 0.1351 & 0.0081 & 0.0214 & 0.0003 & 20.47 & 155.54 & 128.69 & 7.23 & 136.61 & 1.97 \\
\hline ZZO7-11 & 145 & 201 & 0.72 & 0.0491 & 0.0037 & 0.1481 & 0.0097 & 0.0225 & 0.0005 & 150.09 & 170.35 & 140.23 & 8.60 & 143.69 & 3.15 \\
\hline ZZ07-12 & 318 & 609 & 0.52 & 0.0464 & 0.0022 & 0.1424 & 0.0062 & 0.0225 & 0.0003 & 16.77 & 111.10 & 135.20 & 5.55 & 143.25 & 1.81 \\
\hline ZZO7-13 & 166 & 419 & 0.40 & 0.0526 & 0.0027 & 0.1576 & 0.0078 & 0.0217 & 0.0003 & 309.32 & 114.80 & 148.62 & 6.86 & 138.26 & 1.82 \\
\hline ZZ07-14 & 113 & 244 & 0.46 & 0.0480 & 0.0038 & 0.1414 & 0.0098 & 0.0217 & 0.0004 & 98.24 & 177.75 & 134.27 & 8.70 & 138.61 & 2.37 \\
\hline ZZ07-15 & 182 & 323 & 0.56 & 0.0525 & 0.0033 & 0.1542 & 0.0091 & 0.0216 & 0.0003 & 309.32 & 144.43 & 145.62 & 8.05 & 137.93 & 2.17 \\
\hline ZZ07-16 & 242 & 367 & 0.66 & 0.0511 & 0.0031 & 0.1514 & 0.0085 & 0.0217 & 0.0003 & 242.66 & 138.87 & 143.15 & 7.53 & 138.34 & 2.06 \\
\hline ZZ07-17 & 88.5 & 209 & 0.42 & 0.0534 & 0.0051 & 0.1563 & 0.0125 & 0.0220 & 0.0004 & 346.35 & 212.01 & 147.42 & 11.01 & 140.11 & 2.70 \\
\hline ZZ07-18 & 90.7 & 178 & 0.51 & 0.0505 & 0.0039 & 0.1481 & 0.0100 & 0.0217 & 0.0004 & 216.74 & 176.83 & 140.24 & 8.83 & 138.40 & 2.84 \\
\hline ZZ07-19 & 446 & 865 & 0.52 & 0.0481 & 0.0024 & 0.1469 & 0.0071 & 0.0220 & 0.0003 & 105.65 & 111.10 & 139.14 & 6.30 & 140.17 & 1.69 \\
\hline ZZ07-20 & 228 & 325 & 0.70 & 0.0542 & 0.0038 & 0.1543 & 0.0103 & 0.0210 & 0.0004 & 388.94 & 159.24 & 145.74 & 9.05 & 134.03 & 2.38 \\
\hline ZZ07-21 & 82.3 & 179 & 0.46 & 0.0500 & 0.0037 & 0.1538 & 0.0104 & 0.0224 & 0.0004 & 194.53 & 176.83 & 145.25 & 9.17 & 143.03 & 2.43 \\
\hline ZZ07-22 & 76.1 & 159 & 0.48 & 0.0510 & 0.0042 & 0.1441 & 0.0101 & 0.0212 & 0.0004 & 242.66 & 197.20 & 136.72 & 8.95 & 135.37 & 2.72 \\
\hline ZZ07-23 & 133 & 269 & 0.49 & 0.0510 & 0.0038 & 0.1453 & 0.0101 & 0.0208 & 0.0003 & 238.96 & 204.61 & 137.76 & 8.93 & 132.85 & 2.02 \\
\hline ZZ07-24 & 99.6 & 224 & 0.45 & 0.0546 & 0.0038 & 0.1565 & 0.0086 & 0.0213 & 0.0004 & 398.20 & 155.54 & 147.66 & 7.59 & 136.01 & 2.61 \\
\hline ZZ07-26 & 245 & 428 & 0.57 & 0.0528 & 0.0031 & 0.1599 & 0.0093 & 0.0221 & 0.0003 & 320.43 & 130.54 & 150.58 & 8.17 & 140.97 & 2.15 \\
\hline ZZ07-27 & 78.9 & 175 & 0.45 & 0.0492 & 0.0045 & 0.1419 & 0.0111 & 0.0211 & 0.0004 & 166.75 & 199.97 & 134.72 & 9.91 & 134.88 & 2.39 \\
\hline ZZ07-28 & 209 & 623 & 0.34 & 0.0470 & 0.0021 & 0.1448 & 0.0065 & 0.0223 & 0.0003 & 50.10 & 99.99 & 137.31 & 5.76 & 141.91 & 2.00 \\
\hline ZZ07-29 & 65.8 & 149 & 0.44 & 0.0478 & 0.0044 & 0.1384 & 0.0106 & 0.0217 & 0.0005 & 100.09 & 199.97 & 131.64 & 9.42 & 138.37 & 2.95 \\
\hline ZZ07-30 & 90.9 & 168 & 0.54 & 0.0535 & 0.0043 & 0.1626 & 0.0118 & 0.0223 & 0.0004 & 350.06 & 178.68 & 152.95 & 10.30 & 142.34 & 2.78 \\
\hline ZZ11-01 & 144 & 94.5 & 1.53 & 0.0469 & 0.0065 & 0.0919 & 0.0101 & 0.0150 & 0.0004 & 55.65 & 294.41 & 89.24 & 9.38 & 95.68 & 2.50 \\
\hline ZZ11-02 & 837 & 1124 & 0.75 & 0.0482 & 0.0020 & 0.1014 & 0.0043 & 0.0151 & 0.0002 & 109.35 & 98.14 & 98.03 & 3.93 & 96.73 & 1.22 \\
\hline ZZ11-03 & 105 & 104 & 1.01 & 0.0540 & 0.0074 & 0.1019 & 0.0094 & 0.0147 & 0.0005 & 372.28 & 311.07 & 98.48 & 8.69 & 93.92 & 2.95 \\
\hline ZZ11-04 & 268 & 352 & 0.76 & 0.0499 & 0.0032 & 0.1007 & 0.0058 & 0.0145 & 0.0002 & 190.82 & 148.13 & 97.39 & 5.40 & 92.83 & 1.45 \\
\hline ZZ11-05 & 343 & 407 & 0.84 & 0.0507 & 0.0030 & 0.1017 & 0.0058 & 0.0144 & 0.0002 & 227.85 & 141.65 & 98.31 & 5.33 & 92.46 & 1.18 \\
\hline ZZ11-06 & 480 & 532 & 0.90 & 0.0493 & 0.0029 & 0.0998 & 0.0054 & 0.0146 & 0.0002 & 166.75 & 143.50 & 96.59 & 4.98 & 93.53 & 1.27 \\
\hline ZZ11-07 & 58.7 & 61.0 & 0.96 & 0.0546 & 0.0083 & 0.0993 & 0.0110 & 0.0146 & 0.0005 & 394.50 & 344.40 & 96.11 & 10.16 & 93.41 & 3.25 \\
\hline ZZ11-08 & 178 & 228 & 0.78 & 0.0518 & 0.0045 & 0.1025 & 0.0072 & 0.0146 & 0.0003 & 275.99 & 201.83 & 99.10 & 6.59 & 93.55 & 1.71 \\
\hline ZZ11-10 & 187 & 290 & 0.65 & 0.0471 & 0.0041 & 0.0954 & 0.0074 & 0.0150 & 0.0003 & 53.80 & 196.27 & 92.49 & 6.86 & 95.78 & 1.63 \\
\hline ZZ11-11 & 169 & 266 & 0.63 & 0.0499 & 0.0043 & 0.1003 & 0.0075 & 0.0149 & 0.0003 & 190.82 & 198.12 & 97.03 & 6.95 & 95.51 & 1.95 \\
\hline ZZ11-12 & 281 & 308 & 0.91 & 0.0529 & 0.0031 & 0.1099 & 0.0063 & 0.0150 & 0.0003 & 324.13 & 135.17 & 105.86 & 5.74 & 95.81 & 1.75 \\
\hline ZZ11-13 & 187 & 222 & 0.84 & 0.0501 & 0.0044 & 0.1034 & 0.0084 & 0.0152 & 0.0003 & 198.23 & 192.56 & 99.95 & 7.69 & 97.31 & 1.97 \\
\hline
\end{tabular}


TABLE 1 | (Continued) Zircon U-Pb data of granites in the study area.

\begin{tabular}{|c|c|c|c|c|c|c|c|c|c|c|c|c|c|c|c|}
\hline \multirow[t]{2}{*}{ Spot } & \multicolumn{3}{|c|}{ Element content } & \multicolumn{6}{|c|}{ Isotope ratio } & \multicolumn{6}{|c|}{ Age (Ma) } \\
\hline & ${ }^{232} \mathrm{Th}$ & ${ }^{238} \mathrm{U}$ & $\begin{array}{c}\text { Th/ } \\
\mathbf{U}\end{array}$ & $\begin{array}{l}{ }^{207} \mathrm{~Pb} / \\
{ }^{206} \mathrm{~Pb}\end{array}$ & 1sigma & $\begin{array}{c}{ }^{207} \mathrm{~Pb} / \\
{ }^{235} \mathrm{U}\end{array}$ & 1sigma & $\begin{array}{c}{ }^{206} \mathrm{~Pb} / \\
{ }^{238} \mathrm{U}\end{array}$ & 1sigma & $\begin{array}{l}{ }^{207} \mathrm{~Pb} / \\
{ }^{206} \mathrm{~Pb}\end{array}$ & 1sigma & $\begin{array}{c}{ }^{207} \mathrm{~Pb} / \\
{ }^{235} \mathrm{U}\end{array}$ & 1sigma & $\begin{array}{c}{ }^{206} \mathrm{~Pb} / \\
{ }^{238} \mathrm{U}\end{array}$ & 1sigma \\
\hline ZZ11-14 & 406 & 378 & 1.07 & 0.0501 & 0.0038 & 0.1021 & 0.0069 & 0.0149 & 0.0002 & 198.23 & 175.91 & 98.68 & 6.33 & 95.39 & 1.45 \\
\hline ZZ11-18 & 436 & 422 & 1.03 & 0.0483 & 0.0028 & 0.1003 & 0.0055 & 0.0152 & 0.0002 & 122.31 & 133.31 & 97.09 & 5.11 & 97.01 & 1.51 \\
\hline ZZ11-19 & 306 & 368 & 0.83 & 0.0517 & 0.0033 & 0.1018 & 0.0056 & 0.0145 & 0.0002 & 272.29 & 146.28 & 98.48 & 5.13 & 92.67 & 1.35 \\
\hline ZZ11-20 & 211 & 199 & 1.06 & 0.0490 & 0.0043 & 0.0976 & 0.0061 & 0.0149 & 0.0003 & 146.38 & 192.57 & 94.60 & 5.68 & 95.46 & 1.87 \\
\hline ZZ11-21 & 180 & 179 & 1.01 & 0.0542 & 0.0051 & 0.1038 & 0.0074 & 0.0145 & 0.0003 & 375.98 & 212.94 & 100.25 & 6.82 & 92.92 & 1.90 \\
\hline ZZ11-22 & 204 & 277 & 0.74 & 0.0522 & 0.0040 & 0.1051 & 0.0070 & 0.0150 & 0.0003 & 294.51 & 169.42 & 101.50 & 6.45 & 95.88 & 1.75 \\
\hline ZZ11-23 & 212 & 308 & 0.69 & 0.0482 & 0.0033 & 0.0988 & 0.0061 & 0.0150 & 0.0002 & 109.35 & 151.83 & 95.62 & 5.60 & 96.23 & 1.56 \\
\hline ZZ11-24 & 152 & 140 & 1.09 & 0.0563 & 0.0065 & 0.1051 & 0.0091 & 0.0145 & 0.0003 & 464.86 & 259.22 & 101.50 & 8.39 & 92.76 & 2.20 \\
\hline ZZ11-26 & 179 & 187 & 0.96 & 0.0463 & 0.0040 & 0.0918 & 0.0062 & 0.0147 & 0.0003 & 13.06 & 196.27 & 89.20 & 5.79 & 94.08 & 1.90 \\
\hline ZZ11-27 & 149 & 239 & 0.62 & 0.0503 & 0.0035 & 0.1054 & 0.0065 & 0.0152 & 0.0003 & 209.33 & 161.09 & 101.71 & 5.95 & 97.12 & 1.77 \\
\hline ZZ11-28 & 152 & 218 & 0.70 & 0.0504 & 0.0043 & 0.0966 & 0.0068 & 0.0145 & 0.0003 & 213.04 & 0.92 & 93.66 & 6.31 & 92.71 & 1.61 \\
\hline ZZ11-29 & 253 & 374 & 0.68 & 0.0510 & 0.0038 & 0.1020 & 0.0067 & 0.0148 & 0.0003 & 238.96 & 204.61 & 98.66 & 6.17 & 94.69 & 1.82 \\
\hline ZZ11-30 & 222 & 333 & 0.67 & 0.0492 & 0.0041 & 0.0978 & 0.0069 & 0.0149 & 0.0002 & 166.75 & 172.20 & 94.71 & 6.35 & 95.25 & 1.50 \\
\hline HZ05-01 & 128 & 186 & 0.69 & 0.0475 & 0.0041 & 0.1323 & 0.0104 & 0.0204 & 0.0004 & 76.02 & 257.37 & 126.12 & 9.29 & 130.46 & 2.45 \\
\hline HZO5-02 & 114 & 146 & 0.78 & 0.0522 & 0.0048 & 0.1477 & 0.0112 & 0.0205 & 0.0004 & 294.51 & 213.86 & 139.85 & 9.89 & 130.66 & 2.82 \\
\hline HZO5-03 & 534 & 913 & 0.59 & 0.0526 & 0.0019 & 0.1558 & 0.0056 & 0.0214 & 0.0002 & 322.28 & 80.55 & 147.01 & 4.90 & 136.72 & 1.56 \\
\hline HZO5-04 & 227 & 548 & 0.41 & 0.0540 & 0.0032 & 0.1574 & 0.0075 & 0.0211 & 0.0003 & 368.57 & 133.32 & 148.40 & 6.56 & 134.69 & 1.75 \\
\hline HZO5-05 & 159 & 306 & 0.52 & 0.0505 & 0.0036 & 0.1474 & 0.0096 & 0.0213 & 0.0003 & 220.44 & 160.17 & 139.61 & 8.46 & 135.68 & 2.06 \\
\hline HZO5-06 & 205 & 246 & 0.83 & 0.0536 & 0.0045 & 0.1513 & 0.0108 & 0.0209 & 0.0004 & 366.72 & 216.64 & 143.06 & 9.49 & 133.53 & 2.53 \\
\hline HZO5-07 & 159 & 347 & 0.46 & 0.0493 & 0.0033 & 0.1435 & 0.0087 & 0.0214 & 0.0004 & 164.90 & 157.38 & 136.17 & 7.71 & 136.80 & 2.28 \\
\hline HZ05-08 & 107 & 260 & 0.41 & 0.0514 & 0.0047 & 0.1519 & 0.0114 & 0.0220 & 0.0004 & 257.47 & 209.24 & 143.57 & 10.08 & 140.06 & 2.69 \\
\hline HZO5-09 & 65.9 & 88.3 & 0.75 & 0.0501 & 0.0068 & 0.1340 & 0.0110 & 0.0219 & 0.0006 & 198.23 & 288.85 & 127.69 & 9.82 & 139.58 & 3.94 \\
\hline HZO5-10 & 152 & 243 & 0.62 & 0.0493 & 0.0038 & 0.1392 & 0.0097 & 0.0208 & 0.0004 & 161.20 & 183.31 & 132.35 & 8.63 & 132.47 & 2.62 \\
\hline HZO5-11 & 328 & 732 & & & & & & & & & & & & & 1.65 \\
\hline HZO5-12 & 125 & 217 & 0.58 & 0.0461 & 0.0036 & 0.1267 & 0.0092 & 0.0204 & 0.0004 & 400.05 & 211.08 & 121.12 & 8.28 & 130.49 & 2.48 \\
\hline HZO5-13 & 228 & 582 & 0.39 & 0.0484 & 0.0024 & 0.1384 & 0.0066 & 0.0207 & 0.0003 & 120.46 & 86.10 & 131.61 & 5.89 & 132.15 & 1.59 \\
\hline HZO5-14 & 108 & 243 & 0.45 & 0.0526 & 0.0035 & 0.1510 & 0.0092 & 0.0209 & 0.0004 & 322.28 & 151.83 & 142.79 & 8.16 & & 2.32 \\
\hline HZO5-15 & 158 & 202 & 0.78 & 0.0483 & 0.0043 & 0.1348 & 0.0100 & 0.0209 & 0.0005 & 122.31 & 199.97 & 128.42 & 8.96 & 133.37 & 2.85 \\
\hline HZO5-16 & 195 & 390 & 0.50 & 0.0486 & 0.0029 & 0.1408 & 0.0085 & 0.0208 & 0.0003 & 127.87 & 137.02 & 133.75 & 7.55 & 132.97 & 1.90 \\
\hline HZO5-17 & 245 & 594 & 0.41 & 0.0512 & 0.0032 & 0.1535 & 0.0086 & 0.0221 & 0.0003 & 250.07 & 144.43 & 144.96 & 7.56 & 140.60 & 1.95 \\
\hline HZO5-18 & 157 & 259 & 0.61 & 0.0538 & 0.0042 & 0.1498 & 0.0113 & 0.0204 & 0.0004 & 364.87 & 177.76 & 141.74 & 9.94 & 130.37 & 2.43 \\
\hline HZO5-19 & 114 & 303 & 0.38 & 0.0530 & 0.0036 & 0.1461 & 0.0090 & 0.0204 & 0.0003 & 327.84 & 153.68 & 138.49 & 7.99 & 130.10 & 2.06 \\
\hline HZO5-20 & 102 & 167 & 0.61 & 0.0553 & 0.0053 & 0.1483 & 0.0107 & 0.0205 & 0.0005 & 433.38 & 219.41 & 140.44 & 9.45 & 130.83 & 2.90 \\
\hline HZO5-21 & 448 & 570 & 0.79 & 0.0495 & 0.0026 & 0.1512 & 0.0077 & 0.0222 & 0.0003 & 172.31 & 115.73 & 142.94 & 6.79 & 141.37 & 1.85 \\
\hline HZO5-22 & 140 & 260 & 0.54 & 0.0486 & 0.0030 & 0.1483 & 0.0083 & 0.0220 & 0.0004 & 127.87 & 137.02 & 140.41 & 7.31 & 140.18 & 2.42 \\
\hline HZO5-23 & 154 & 308 & 0.50 & 0.0512 & 0.0032 & 0.1506 & 0.0081 & 0.0219 & 0.0004 & 250.07 & 142.58 & 142.43 & 7.16 & 139.94 & 2.48 \\
\hline HZO5-24 & 139 & 282 & 0.49 & 0.0517 & 0.0035 & 0.1483 & 0.0089 & 0.0214 & 0.0003 & 272.29 & 149.06 & 140.37 & 7.89 & 136.21 & 2.08 \\
\hline HZO5-25 & 56.2 & 90.4 & 0.62 & 0.0534 & 0.0057 & 0.1544 & 0.0133 & 0.0212 & 0.0005 & 342.65 & 244.42 & 145.80 & 11.66 & 135.52 & 3.46 \\
\hline HZO5-26 & 174 & 176 & 0.99 & 0.0552 & 0.0040 & 0.1582 & 0.0092 & 0.0214 & 0.0004 & 420.42 & 160.17 & 149.16 & 8.04 & 136.71 & 2.54 \\
\hline HZO5-27 & 105 & 201 & 0.53 & 0.0513 & 0.0035 & 0.1544 & 0.0091 & 0.0218 & 0.0004 & 253.77 & 183.31 & 145.77 & 7.99 & 138.92 & 2.36 \\
\hline HZO5-28 & 127 & 214 & 0.60 & 0.0512 & 0.0037 & 0.1446 & 0.0092 & 0.0209 & 0.0004 & 255.62 & 164.79 & 137.15 & 8.20 & 133.20 & 2.43 \\
\hline HZO5-29 & 129 & 386 & 0.33 & 0.0520 & 0.0032 & 0.1525 & 0.0092 & 0.0214 & 0.0003 & 283.40 & 110.17 & 144.12 & 8.13 & 136.59 & 1.93 \\
\hline HZO5-30 & 97.9 & 184 & 0.53 & 0.0490 & 0.0038 & 0.1425 & 0.0088 & 0.0214 & 0.0004 & 150.09 & 174.05 & 135.27 & 7.82 & 136.71 & 2.40 \\
\hline
\end{tabular}

concentrations of Th (50.5-318 ppm) and U (149-865 ppm), corresponding $\mathrm{Th} / \mathrm{U}$ ratios of $0.34-0.72$, showing the characteristic of magmatic zircon. These data yield a weighted mean ${ }^{206} \mathrm{~Pb} /{ }^{238} \mathrm{U}$ age of $138.4 \pm 1.1 \mathrm{Ma}$ (MSWD = 1.6), representing the formation age of the granite. A total of 25 valid data were obtained from ZZ11. These zircon grains have moderate and variable concentrations of Th (58.7-837 ppm) and $U$ (61-1,124 ppm), corresponding $\mathrm{Th} / \mathrm{U}$ ratios of $0.62-1.53$, showing the characteristic of magmatic zircon. These data yield a weighted mean ${ }^{206} \mathrm{~Pb} /{ }^{238} \mathrm{U}$ age of $94.7 \pm 0.25 \mathrm{Ma}(\mathrm{MSWD}=1.01$ ), representing the formation age of the granite. A total of 30 valid data were obtained from HZ05. These zircon grains have moderate and variable concentrations of $\mathrm{Th}$ (56.2-448 ppm) and $\mathrm{U}$
(88.3-548 ppm), corresponding Th/U ratios of 0.33-0.99, showing the characteristic of magmatic zircon. These data yield a weighted mean ${ }^{206} \mathrm{~Pb} /{ }^{238} \mathrm{U}$ age of $135.1 \pm 1.3 \mathrm{Ma}(\mathrm{MSWD}=2.4)$, representing the formation age of the granite.

There is no obvious pattern of age distribution of such samples in the region. In general, the age of all samples in the study area shows that they are formed in Cretaceous age, indicating that Yanshanian tectonic thermal events are the dominant thermal events in the area. According to the $\mathrm{U}-\mathrm{Pb}$ dating results, at least three acidic magmatic events occurred during Cretaceous: early stage of Early Cretaceous (about 138-135.1 Ma), late stage of Early Cretaceous (110-121 Ma) and the early stage of Late Cretaceous (about 93-96 Ma). 


\section{Geochemistry}

20 fresh granite samples are obtained for major and trace element analyses. The results are listed in Table 2 . The samples have very low loss on ignition $(\sim 0.85 \mathrm{wt} \%)$, indicating little or no modification by weathering. Rock geochemical test results show that the rock mass geochemical composition analyses are as follows: $\mathrm{SiO}_{2}$ content ranged from 65.86 to $83.83 \%$ with an average of $73.64 \%$; $\mathrm{TiO}_{2}$ content ranged from 0.08 to $0.55 \%$ with an average of $0.23 \% ; \mathrm{Al}_{2} \mathrm{O}_{3}$ content ranged from 7.38 to $15.8 \%$ with an average of $13.53 \%$; $\mathrm{MgO}$ content ranged from 0.12 to $1.73 \%$ with an average of $0.48 \%$; and $\left(\mathrm{K}_{2} \mathrm{O}+\mathrm{Na}_{2} \mathrm{O}\right)$ content ranged from 4.76 to $8.87 \%$ with an average of $7.66 \%$, belonging to high-K calc-alkaline series. The granites are characterized by weakly peraluminous $(\mathrm{A} / \mathrm{CNK}=1.03)$. At the diagram of $\mathrm{SiO}_{2}$ $\left(\mathrm{Na}_{2} \mathrm{O}+\mathrm{K}_{2} \mathrm{O}\right)$ (Figure 7), sample dots fell within the granite region. The aluminum saturation index $(\mathrm{A} / \mathrm{CNK})$ ranges from 0.88 to 1.33 and ranges mainly from 1.0 to 1.1 on the diagram of $\mathrm{A} / \mathrm{NK}-\mathrm{A} / \mathrm{CNK}$ (Figure 7).

On the primitive mantle normalized trace element spider diagram (Figure 8), Large ionic Lithophile Elements (LILE) such as $K$ and $R b$, Th, and $U$ are enriched, and there is a strong loss of $\mathrm{Ba}, \mathrm{Sr}$, and High Field Strong Elements (HFSE) such as $\mathrm{Nb}, \mathrm{Ti}, \mathrm{Ta}$, and $\mathrm{P}$, which are similar to the geochemical characteristics of rocks in the subduction zone (Kelemen et al., 2003). On the Chondrite Normalized REE distribution diagram (Figure 8), the pattern is smooth and show light rare Earth element (LREE) concentration and heavy rare Earth element (HREE) losses. $\mathrm{Eu}$ in the pattern shows negative anomaly, indicating that the magma evolution of plagioclase crystallization separation. The value of $\Sigma$ REE is between 50.50 $\times 10^{-6}-401.02 \times 10^{-6}$, with a mean of $183.31 \times 10^{-6}$. The value of LREE/HREE is $0.84-12.46$, with an average of 6.44 , showing that obvious fractionation between light and heavy rare Earth. The value of $(\mathrm{La} / \mathrm{Yb})_{\mathrm{N}}$ is $0.67-19.24$, while the average value is 7.18 . The value of Eu is between 0.03 and 0.71 , and the average value is 0.32. In primitive mantle normalized trace element spider diagram, most of the sample elements distribution features are similar, indicating that most of the samples have the same or similar formation patterns.

\section{DISCUSSION}

\section{Genetic Mechanism of Yanshannian Granite}

Large-scale Yanshanian granites constitute the main part of southeastern China, and were formed related to the subduction of the Pacific plate, large-scale basaltic magma invasion, crust-mantle mixing, and extensive melting of crustal rocks (Liu et al., 2016; Pan et al., 2017). Meanwhile, other researchers have proposed the relationship between the lithospheric thinning in eastern China and the subduction of the Pacific plate (Wang and Shen, 2003; Wu et al., 2007). Li et al. (2017) discussed the starting time of palaeo-Pacific subduction. Through the study of Mesozoic tectonic transition and transformation in South China, they found that the palaeoPacific plate subduction had already started in the Late Triassic. It is considered that the crustal thickness of the South
China Block gradually thinning from west to east is caused by two crustal mantle interactions, which resulted in two lithospheric delamination and subsidence, and both of them are related to the subduction of the palaeo-Pacific Plate, which were caused by plate tearing, subduction retraction and high Angle subduction respectively.

According to the results of this paper, at least three acidic magmatic events occurred during Cretaceous: early stage of Early Cretaceous (about 138-135.1 Ma), late stage of Early Cretaceous (about 112-121 Ma) and the early stage of Late Cretaceous (about 93-96 Ma).

The age of the granite sample (ZZ07) in Zhangzhou area and the granite sample (HZ05) in the south of Huangshadong geothermal field in Huizhou are $138.4 \pm 1.1 \mathrm{Ma}$ and $135.1 \pm$ 1.3 Ma, indicating that the granite body is the product of magmatic activity in the early stage of early Cretaceous. At the diagram of $\mathrm{SiO}_{2} /\left(\mathrm{Na}_{2} \mathrm{O}+\mathrm{K}_{2} \mathrm{O}\right.$ ) (Figure 7A), sample dots fell within the granite region. The granites are characterized by weakly peraluminous $(\mathrm{A} / \mathrm{CNK}=1.02-1.08)$ (Figure $7 \mathrm{~B})$. The aluminum saturation index $(\mathrm{A} / \mathrm{CNK})$ ranges from 1.14 to 1.33 . On the $\mathrm{SiO}_{2}-\mathrm{FeO}_{\mathrm{T}} /\left(\mathrm{FeO}_{\mathrm{T}}+\mathrm{MgO}\right)$ and $\mathrm{SiO}_{2}-\mathrm{Al}_{2} \mathrm{O}_{3}$ diagram (Figures 9A,B), most samples are plotted in the POG (postOrogenic Grabite) zone (Frost et al., 2001). On the Nb-Y and (Y + $\mathrm{Nb}$ )-Rb diagram (Figures 9C,D), samples are plotted within the scope of the volcanic island arc granite (VAG), within plate granite (WPG) and sys-collision granite (syn-COLG). Combined with the trace element characteristics of the samples, it can be inferred that the samples have dual characteristics of volcanic arc and within plate environment. The ages of the granite rock (GA02, QQ02) and the granite rock (ZZ02) around the Fuzhou geothermal field and Zhangzhou geothermal field are $117.9 \pm 0.47 \mathrm{Ma}, 112.9 \pm 0.85 \mathrm{Ma}$ and $121.3 \pm$ 1.2 Ma, respectively, which are the products of magmatic activity in the late Early Cretaceous. It is the product of the same magmatism as most of the granites along the Fujian coast area in Fujian Province. At the diagram of $\mathrm{SiO}_{2} /\left(\mathrm{Na}_{2} \mathrm{O}+\mathrm{K}_{2} \mathrm{O}\right)$, sample dots fell within the granodiorite region (Figure 7A). They are characterized by Metaluminous - peraluminous $(\mathrm{A} / \mathrm{CNK}=$ 0.95-1.05) (Figure 7B). The aluminum saturation index (A/ $\mathrm{CNK})$ ranges from 1.42 to 1.68 . On the $\mathrm{SiO}_{2}-\mathrm{FeO}_{\mathrm{T}} /\left(\mathrm{FeO}_{\mathrm{T}}+\right.$ $\mathrm{MgO}$ ) and $\mathrm{SiO}_{2}-\mathrm{Al}_{2} \mathrm{O}_{3}$ diagram (Figures 9A,B), most samples are plotted out of the POG (post-Orogenic Grabite) zone (Frost et al., 2001). On the Nb-Y and $(\mathrm{Y}+\mathrm{Nb})-\mathrm{Rb}$ diagram (Figures 9C,D), samples are plotted within the scope of the volcanic island arc granite (VAG), indicating rocks in this periods are mainly influenced by subduction related environment. The age of the sample granite rock mass (Z11) in coastal Zhangzhou is $94.7 \pm$ $0.65 \mathrm{Ma}$, which is similar to the age of the rock mass (93 Ma, $95 \mathrm{Ma}$ ) in the south of Fuzhou geothermal field. It is the product of magmatic activity in the early stage of Late Cretaceous. At the diagram of $\mathrm{SiO}_{2} /\left(\mathrm{Na}_{2} \mathrm{O}+\mathrm{K}_{2} \mathrm{O}\right)$, sample dots fell within the granite region (Figure $7 \mathbf{A}$ ). They are characterized by also weakly peraluminous $(\mathrm{A} / \mathrm{CNK}=1.03-1.34)$ (Figure $7 \mathbf{B})$. The aluminum saturation index $(\mathrm{A} / \mathrm{CNK})$ ranges from 1.06 to 1.38 . On the $\mathrm{SiO}_{2}-\mathrm{FeO}_{\mathrm{T}} /\left(\mathrm{FeO}_{\mathrm{T}}+\mathrm{MgO}\right)$ and $\mathrm{SiO}_{2}-\mathrm{Al}_{2} \mathrm{O}_{3}$ diagram (Figures 9A,B), most samples are plotted in the POG (postOrogenic Grabite) zone (Frost et al., 2001). On the Nb-Y and 
TABLE 2 | Analytical data of major (\%) and trace element(ppm) of granites in the study area.

\begin{tabular}{|c|c|c|c|c|c|c|c|c|c|c|c|c|c|c|c|c|c|c|c|c|}
\hline Sample & GA02 & GA05 & GM01 & GM02 & QQ02 & ZZ01 & ZZ02 & ZZ03 & ZZO4 & ZZ07 & ZZ09 & ZZ11 & ZZ12 & ZZ13 & ZZ14 & ZZ15 & ZZ17 & HZ05 & HZ06 & HZ18 \\
\hline $\mathrm{SiO} 2$ & 66.74 & 65.86 & 73.18 & 74.69 & 75.27 & 68.43 & 69.24 & 69.89 & 69.62 & 73.05 & 83.83 & 73.59 & 77.34 & 75.35 & 76.67 & 75.66 & 76.34 & 75.50 & 75.03 & 77.56 \\
\hline TiO2 & 0.51 & 0.55 & 0.26 & 0.24 & 0.14 & 0.39 & 0.35 & 0.31 & 0.35 & 0.13 & 0.08 & 0.31 & 0.08 & 0.12 & 0.11 & 0.13 & 0.17 & 0.11 & 0.11 & 0.09 \\
\hline Al2O3 & 15.16 & 15.25 & 14.68 & 13.65 & 13.41 & 15.80 & 15.21 & 14.49 & 14.65 & 13.70 & 7.38 & 13.71 & 12.83 & 13.28 & 12.45 & 13.58 & 12.71 & 12.93 & 13.37 & 12.60 \\
\hline Fe2O3T & 4.26 & 4.80 & 1.60 & 1.63 & 1.54 & 3.06 & 2.62 & 2.61 & 2.72 & 1.66 & 0.47 & 2.05 & 0.58 & 0.81 & 0.79 & 0.95 & 1.05 & 1.57 & 1.36 & 0.67 \\
\hline FeOT & 3.83 & 4.32 & 1.44 & 1.47 & 1.39 & 2.75 & 2.36 & 2.34 & 2.45 & 1.50 & 0.43 & 1.84 & 0.52 & 0.73 & 0.71 & 0.85 & 0.95 & 1.42 & 1.22 & 0.60 \\
\hline $\mathrm{MnO}$ & 0.11 & 0.11 & 0.07 & 0.06 & 0.04 & 0.05 & 0.07 & 0.09 & 0.08 & 0.04 & 0.03 & 0.09 & 0.07 & 0.05 & 0.04 & 0.09 & 0.04 & 0.04 & 0.03 & 0.03 \\
\hline MgO & 1.52 & 1.73 & 0.44 & 0.37 & 0.22 & 0.92 & 0.77 & 0.79 & 0.85 & 0.15 & 0.21 & 0.51 & 0.06 & 0.15 & 0.15 & 0.17 & 0.20 & 0.14 & 0.19 & 0.12 \\
\hline $\mathrm{CaO}$ & 3.84 & 3.92 & 1.53 & 1.07 & 1.17 & 3.35 & 2.86 & 2.06 & 2.66 & 1.08 & 1.75 & 0.88 & 0.28 & 0.70 & 0.66 & 0.89 & 0.79 & 0.76 & 0.79 & 0.13 \\
\hline $\mathrm{Na2O}$ & 3.48 & 3.46 & 3.96 & 3.69 & 4.17 & 3.49 & 3.21 & 3.20 & 3.19 & 3.20 & 2.52 & 3.57 & 4.52 & 3.29 & 3.20 & 3.99 & 3.53 & 3.74 & 3.75 & 2.09 \\
\hline K2O & 3.05 & 3.21 & 4.17 & 4.29 & 3.73 & 3.62 & 3.76 & 4.57 & 4.07 & 5.28 & 2.25 & 4.47 & 4.35 & 4.78 & 4.53 & 4.19 & 4.60 & 4.71 & 5.13 & 5.28 \\
\hline P2O5 & 0.16 & 0.18 & 0.05 & 0.05 & 0.04 & 0.14 & 0.10 & 0.10 & 0.11 & 0.03 & 0.02 & 0.08 & 0.01 & 0.03 & 0.02 & 0.03 & 0.03 & 0.03 & 0.02 & 0.01 \\
\hline LOI & 1.01 & 0.65 & 0.38 & 0.41 & 0.43 & 0.54 & 1.35 & 1.42 & 1.41 & 1.38 & 1.66 & 1.01 & 0.28 & 1.48 & 1.00 & 0.44 & 0.14 & 0.45 & 0.43 & 1.10 \\
\hline SUM & 99.83 & 99.72 & 100.31 & 100.15 & 100.17 & 99.80 & 99.53 & 99.51 & 99.70 & 99.70 & 100.18 & 100.26 & 100.39 & 100.02 & 99.62 & 100.11 & 99.61 & 99.97 & 100.20 & 99.68 \\
\hline Li & 7.48 & 14.16 & 22.27 & 14.90 & 12.87 & 5.82 & 7.87 & 6.14 & 8.05 & 7.67 & 3.06 & 12.72 & 29.82 & 6.51 & 6.66 & 32.62 & 10.45 & 31.40 & 27.34 & 11.30 \\
\hline $\mathrm{Be}$ & 1.90 & 1.70 & 2.48 & 2.95 & 2.07 & 2.27 & 2.36 & 2.41 & 2.46 & 3.92 & 0.87 & 3.81 & 5.38 & 2.94 & 2.13 & 1.96 & 4.70 & 6.43 & 4.17 & 4.27 \\
\hline Sc & 8.44 & 10.11 & 4.55 & 4.18 & 5.24 & 4.27 & 5.20 & 4.99 & 7.47 & 2.50 & 0.80 & 4.57 & 1.58 & 2.69 & 2.48 & 3.32 & 1.71 & 5.21 & 5.34 & 2.50 \\
\hline V & 68.35 & 75.21 & 15.12 & 13.15 & 6.29 & 42.70 & 26.92 & 21.09 & 33.17 & 5.87 & 4.73 & 24.17 & 2.00 & 4.21 & 6.10 & 6.10 & 8.91 & 5.19 & 4.20 & 1.53 \\
\hline $\mathrm{Cr}$ & 1.50 & 1.61 & 1.06 & 0.92 & 0.42 & 1.51 & 0.87 & 0.96 & 1.00 & 0.49 & 0.89 & 0.98 & 0.33 & 0.38 & 0.34 & 0.32 & 0.88 & 0.91 & 0.83 & 0.26 \\
\hline Co & 6.84 & 8.07 & 1.26 & 1.31 & 0.90 & 4.98 & 3.77 & 3.31 & 3.41 & 1.03 & 0.77 & 2.13 & 0.07 & 0.54 & 0.39 & 0.53 & 0.68 & 0.56 & 0.41 & 0.10 \\
\hline $\mathrm{Ni}$ & 2.07 & 2.15 & 1.12 & 1.01 & 0.89 & 2.41 & 1.27 & 1.28 & 1.28 & 0.82 & 1.05 & 1.43 & 0.58 & 0.74 & 0.63 & 0.59 & 1.17 & 0.71 & 0.87 & 0.55 \\
\hline $\mathrm{Cu}$ & 3.06 & 3.45 & 1.33 & 1.81 & 0.40 & 2.91 & 58.72 & 16.73 & 60.52 & 0.45 & 0.64 & 1.24 & 0.32 & 0.46 & 0.36 & 0.45 & 0.98 & 0.34 & 0.44 & 0.71 \\
\hline $\mathrm{Zn}$ & 52.38 & 47.76 & 36.14 & 40.53 & 35.11 & 76.11 & 48.94 & 49.04 & 33.91 & 25.69 & 6.19 & 50.24 & 39.48 & 21.08 & 17.89 & 26.62 & 14.54 & 17.29 & 22.26 & 36.71 \\
\hline Ga & 15.30 & 15.76 & 16.70 & 16.03 & 15.85 & 18.90 & 15.10 & 12.72 & 15.65 & 17.38 & 7.16 & 17.65 & 19.23 & 27.27 & 25.91 & 15.10 & 15.77 & 17.97 & 17.56 & 16.93 \\
\hline $\mathrm{Rb}$ & 90.99 & 112.01 & 170.09 & 155.20 & 133.37 & 133.14 & 116.49 & 144.05 & 131.38 & 174.51 & 62.06 & 210.19 & 267.52 & 191.46 & 179.68 & 189.25 & 233.26 & 262.13 & 263.46 & 324.54 \\
\hline $\mathrm{Sr}$ & 431.06 & 415.98 & 212.04 & 209.11 & 156.40 & 527.36 & 392.22 & 303.69 & 383.07 & 150.88 & 76.96 & 154.73 & 5.22 & 1378.40 & 934.19 & 132.30 & 75.50 & 55.80 & 62.44 & 30.99 \\
\hline Y & 15.98 & 21.59 & 27.60 & 29.06 & 29.35 & 13.29 & 21.35 & 24.63 & 31.65 & 36.83 & 18.59 & 30.76 & 29.91 & 19.15 & 20.32 & 25.91 & 27.96 & 49.38 & 26.70 & 37.10 \\
\hline $\mathrm{Zr}$ & 169.31 & 183.67 & 189.84 & 174.22 & 144.86 & 162.57 & 183.96 & 168.97 & 186.27 & 125.39 & 74.57 & 186.87 & 79.85 & 82.43 & 66.27 & 86.58 & 129.71 & 140.49 & 120.91 & 83.32 \\
\hline $\mathrm{Nb}$ & 9.12 & 9.97 & 15.77 & 16.33 & 13.59 & 9.25 & 12.02 & 11.56 & 15.66 & 11.47 & 5.77 & 22.01 & 37.09 & 15.50 & 13.71 & 21.89 & 23.34 & 26.17 & 17.96 & 25.55 \\
\hline Sn & 1.08 & 1.36 & 2.07 & 2.20 & 2.02 & 1.19 & 1.73 & 1.78 & 2.72 & 2.53 & 1.03 & 2.89 & 2.98 & 1.95 & 1.82 & 2.58 & 1.36 & 2.93 & 2.82 & 1.56 \\
\hline Cs & 2.87 & 2.57 & 5.56 & 3.72 & 2.04 & 5.10 & 2.29 & 2.28 & 2.15 & 1.24 & 0.52 & 3.42 & 6.92 & 15.89 & 11.50 & 3.65 & 2.93 & 7.34 & 6.28 & 4.07 \\
\hline $\mathrm{Ba}$ & 987.77 & 975.04 & 1207.11 & 1068.56 & 838.01 & 844.17 & 558.96 & 678.02 & 580.46 & 647.35 & 375.27 & 418.07 & 16.66 & 1479.56 & 1122.94 & 694.20 & 203.27 & 140.57 & 167.22 & 97.67 \\
\hline La & 34.94 & 42.64 & 55.29 & 52.86 & 30.36 & 34.59 & 36.67 & 39.59 & 45.19 & 49.08 & 19.65 & 47.87 & 21.88 & 24.55 & 22.60 & 29.54 & 39.57 & 42.38 & 25.79 & 115.84 \\
\hline $\mathrm{Ce}$ & 63.02 & 77.08 & 105.87 & 101.44 & 58.86 & 65.44 & 69.14 & 75.19 & 87.19 & 98.22 & 40.22 & 89.59 & 44.37 & 48.88 & 46.18 & 58.76 & 72.62 & 87.62 & 52.83 & 57.85 \\
\hline $\operatorname{Pr}$ & 6.52 & 8.02 & 11.58 & 10.92 & 6.80 & 7.24 & 7.44 & 8.00 & 9.53 & 10.92 & 4.43 & 9.69 & 4.55 & 5.30 & 4.92 & 6.34 & 7.47 & 10.11 & 6.21 & 20.79 \\
\hline $\mathrm{Nd}$ & 23.18 & 28.76 & 41.92 & 39.51 & 25.69 & 26.41 & 26.03 & 28.10 & 33.39 & 38.05 & 15.85 & 32.19 & 13.42 & 18.01 & 16.49 & 21.34 & 23.20 & 36.99 & 22.86 & 60.89 \\
\hline Sm & 4.07 & 5.19 & 7.10 & 7.01 & 5.11 & 4.76 & 4.84 & 5.45 & 6.81 & 7.32 & 3.15 & 6.08 & 3.05 & 3.75 & 3.53 & 4.54 & 4.33 & 8.85 & 5.47 & 10.89 \\
\hline Eu & 1.11 & 1.09 & 1.26 & 1.19 & 0.87 & 1.05 & 1.11 & 1.21 & 1.07 & 0.74 & 0.37 & 0.81 & 0.15 & 0.48 & 0.47 & 0.53 & 0.36 & 0.38 & 0.30 & 1.01 \\
\hline Gd & 3.55 & 4.24 & 5.45 & 5.52 & 4.66 & 3.39 & 3.73 & 4.24 & 5.21 & 5.65 & 2.52 & 4.87 & 2.64 & 2.82 & 2.71 & 3.59 & 3.27 & 7.38 & 4.43 & 8.18 \\
\hline $\mathrm{Tb}$ & 0.53 & 0.59 & 0.80 & 0.80 & 0.74 & 0.47 & 0.57 & 0.68 & 0.86 & 0.97 & 0.46 & 0.81 & 0.57 & 0.52 & 0.51 & 0.66 & 0.60 & 1.33 & 0.75 & 1.08 \\
\hline Dy & 2.99 & 3.52 & 4.60 & 4.78 & 4.48 & 2.54 & 3.59 & 4.12 & 5.13 & 5.75 & 2.93 & 4.86 & 4.01 & 3.14 & 3.19 & 4.08 & 3.75 & 8.19 & 4.57 & 6.00 \\
\hline $\mathrm{Ho}$ & 0.61 & 0.71 & 0.92 & 0.95 & 0.94 & 0.47 & 0.66 & 0.81 & 1.01 & 1.20 & 0.62 & 0.98 & 0.89 & 0.61 & 0.64 & 0.82 & 0.83 & 1.63 & 0.88 & 1.14 \\
\hline Er & 1.78 & 2.10 & 2.82 & 2.86 & 2.85 & 1.33 & 2.16 & 2.41 & 3.03 & 3.52 & 1.82 & 2.94 & 2.97 & 1.86 & 1.87 & 2.44 & 2.67 & 4.74 & 2.43 & 3.44 \\
\hline Tm & 0.27 & 0.32 & 0.39 & 0.43 & 0.45 & 0.17 & 0.32 & 0.38 & 0.46 & 0.54 & 0.29 & 0.44 & 0.47 & 0.30 & 0.30 & 0.38 & 0.45 & 0.71 & 0.37 & 0.54 \\
\hline $\mathrm{Yb}$ & 1.88 & 2.27 & 2.70 & 2.78 & 3.15 & 1.23 & 2.18 & 2.49 & 3.19 & 3.70 & 2.00 & 3.22 & 3.39 & 2.04 & 2.08 & 2.73 & 3.37 & 4.89 & 2.45 & 3.81 \\
\hline Lu & 0.30 & 0.34 & 0.40 & 0.42 & 0.47 & 0.17 & 0.32 & 0.38 & 0.47 & 0.57 & 0.36 & 0.47 & 0.51 & 0.32 & 0.32 & 0.43 & 0.53 & 0.75 & 0.38 & 0.60 \\
\hline $\mathrm{Hf}$ & 4.39 & 4.56 & 5.42 & 5.11 & 4.47 & 4.44 & 4.89 & 4.52 & 5.03 & 4.33 & 2.36 & 5.71 & 4.05 & 2.96 & 2.38 & 3.26 & 4.40 & 5.14 & 4.04 & 3.72 \\
\hline $\mathrm{Ta}$ & 0.66 & 0.70 & 1.08 & 1.08 & 1.00 & 0.65 & 0.82 & 0.85 & 1.22 & 1.58 & 0.46 & 1.51 & 2.07 & 1.39 & 1.21 & 2.22 & 1.93 & 2.25 & 1.45 & 1.53 \\
\hline $\mathrm{Tl}$ & 0.52 & 0.73 & 1.05 & 0.87 & 0.62 & 0.61 & 0.83 & 1.30 & 0.96 & 0.99 & 0.39 & 1.44 & 1.46 & 1.37 & 1.35 & 1.08 & 1.17 & 1.46 & 1.41 & 2.48 \\
\hline $\mathrm{Pb}$ & 22.31 & 17.76 & 34.83 & 33.99 & 16.54 & 23.25 & 25.30 & 19.93 & 16.29 & 21.95 & 3.88 & 32.90 & 36.59 & 26.41 & 26.40 & 26.24 & 28.24 & 19.91 & 21.00 & 14.85 \\
\hline Th & 17.38 & 19.36 & 23.75 & 24.02 & 13.98 & 20.82 & 12.79 & 14.31 & 21.04 & 20.57 & 9.22 & 27.36 & 29.67 & 15.78 & 14.46 & 17.44 & 32.61 & 27.06 & 16.77 & 26.36 \\
\hline U & 3.04 & 3.31 & 3.63 & 4.96 & 2.71 & 4.29 & 3.01 & 2.89 & 3.63 & 4.48 & 1.56 & 4.82 & 8.33 & 3.48 & 3.27 & 7.70 & 4.30 & 7.11 & 4.61 & 3.17 \\
\hline
\end{tabular}



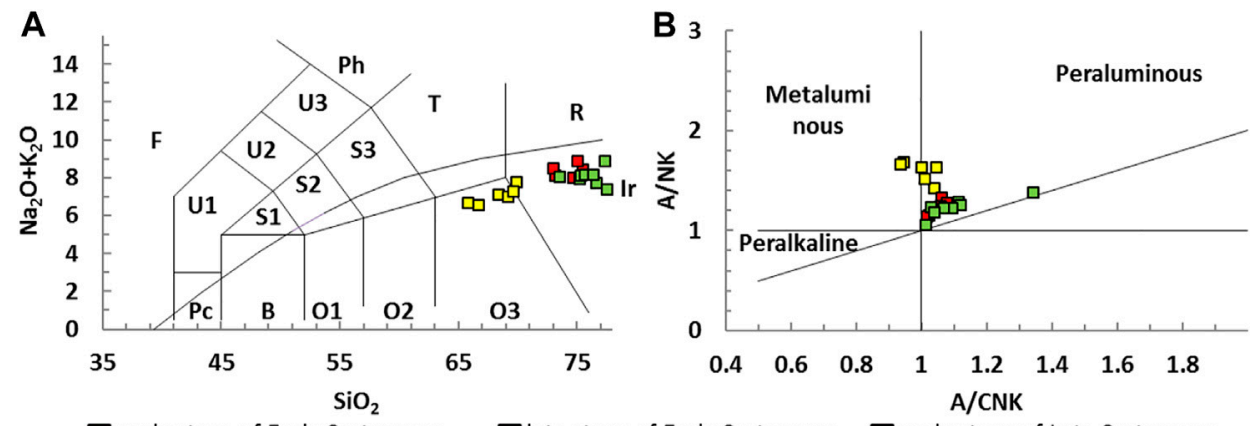

$\square$ early stage of Early Cretaceous

$\square$ late stage of Early Cretaceous

$\square$ early stage of Late Cretaceous

FIGURE 7 | TAS (A) and AVCNK (B) diagrams of magmatic rocks.
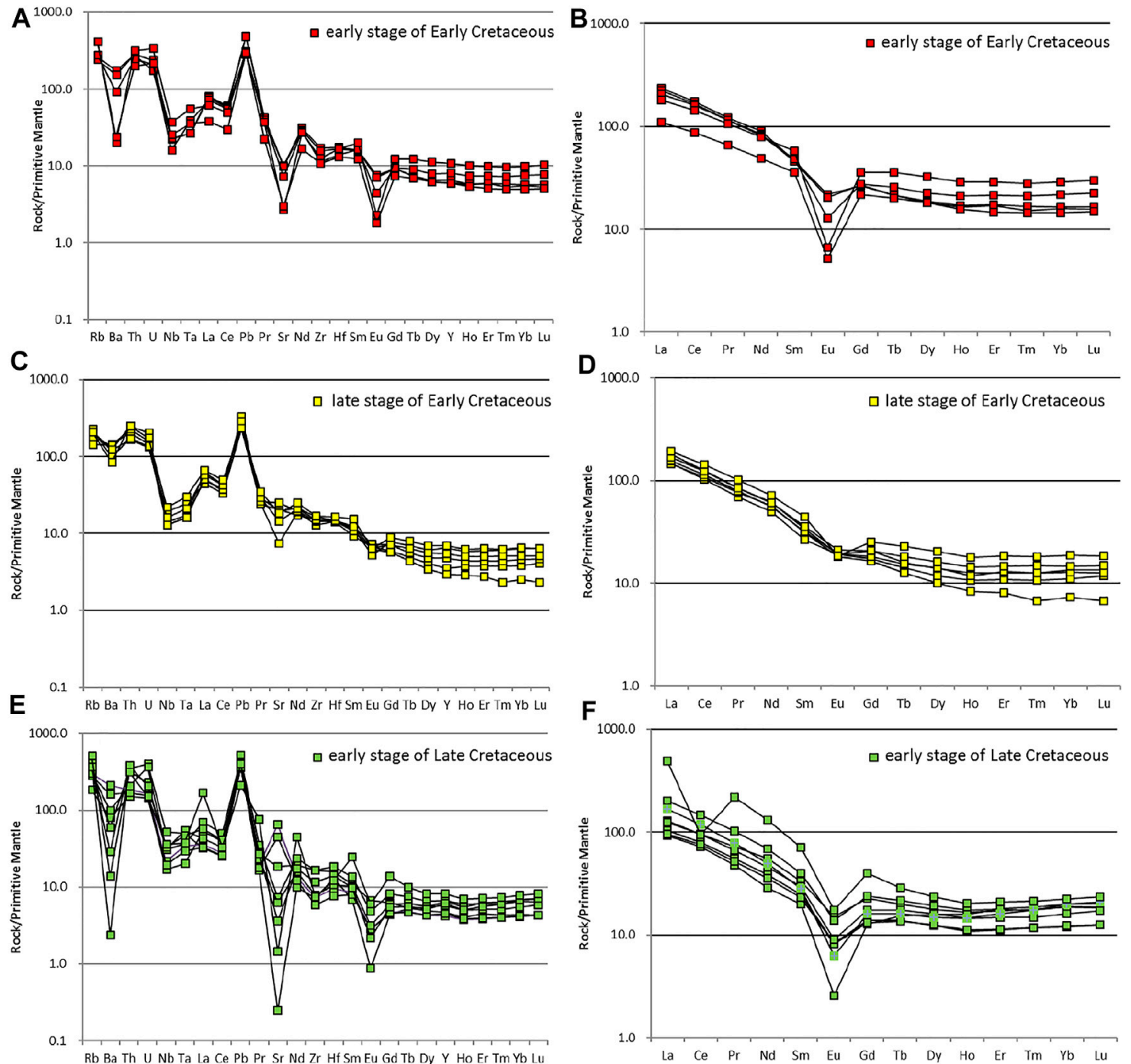

FIGURE 8| Chondrite Normalized REE distribution patterns (A), (C), (E) and primitive mantle normalized trace element spider diagram (B), (D), (F) of granitic rocks. 

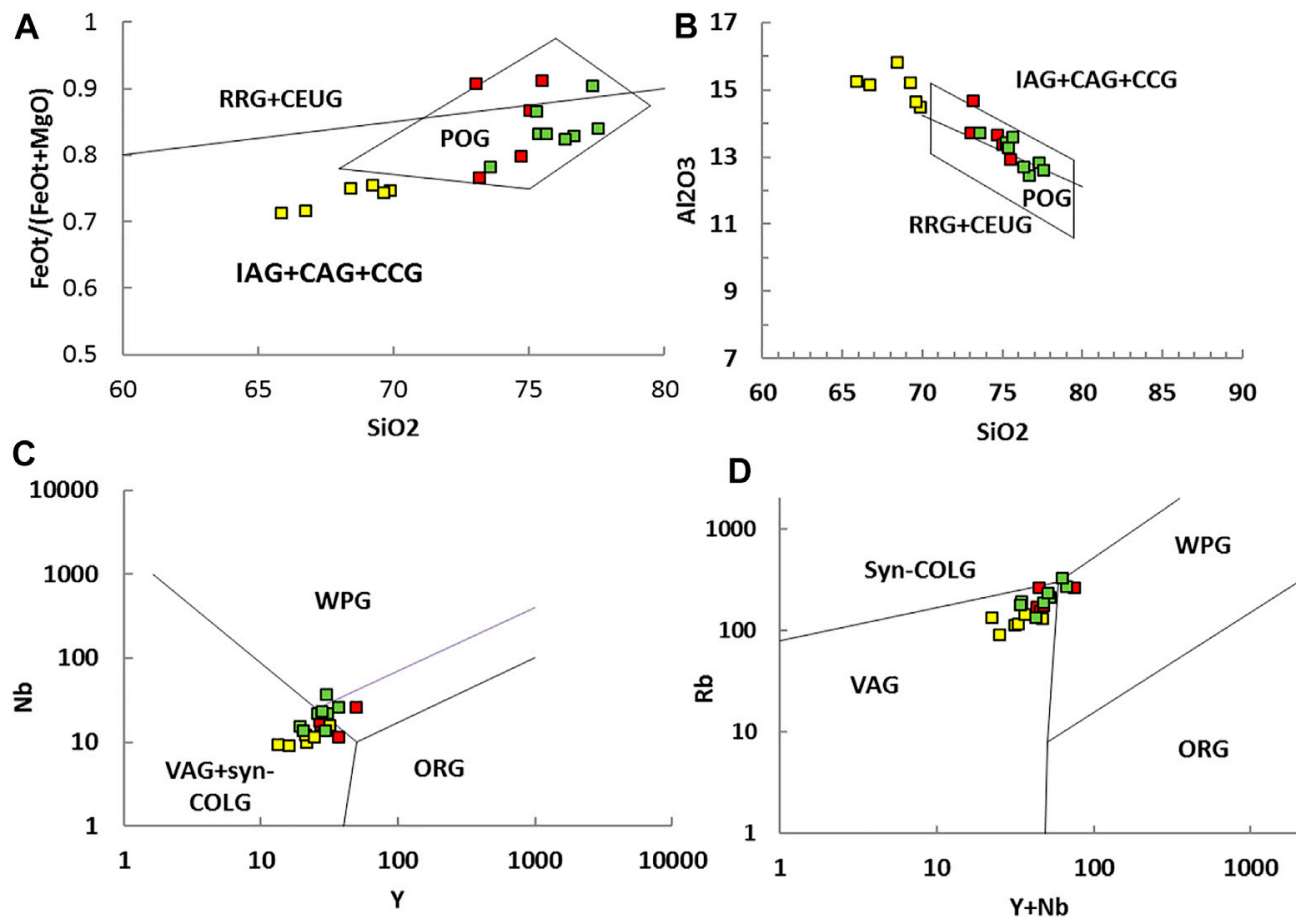

$\square$ early stage of Early Cretaceous $\quad \square$ late stage of Early Cretaceous $\square$ early stage of Late Cretaceous

FIGURE 9 | $\mathrm{SiO}_{2}-\mathrm{FeOT} /(\mathrm{FeOT}+\mathrm{MgO})$, (A) $\mathrm{SiO}_{2}-\mathrm{Al}_{2} \mathrm{O}_{3}$, (B) $\mathrm{Y}-\mathrm{Nb}$, (C) and $(\mathrm{Yb}+\mathrm{Nb})$ - $\mathrm{Rb}$, (D) diagrams of granites.
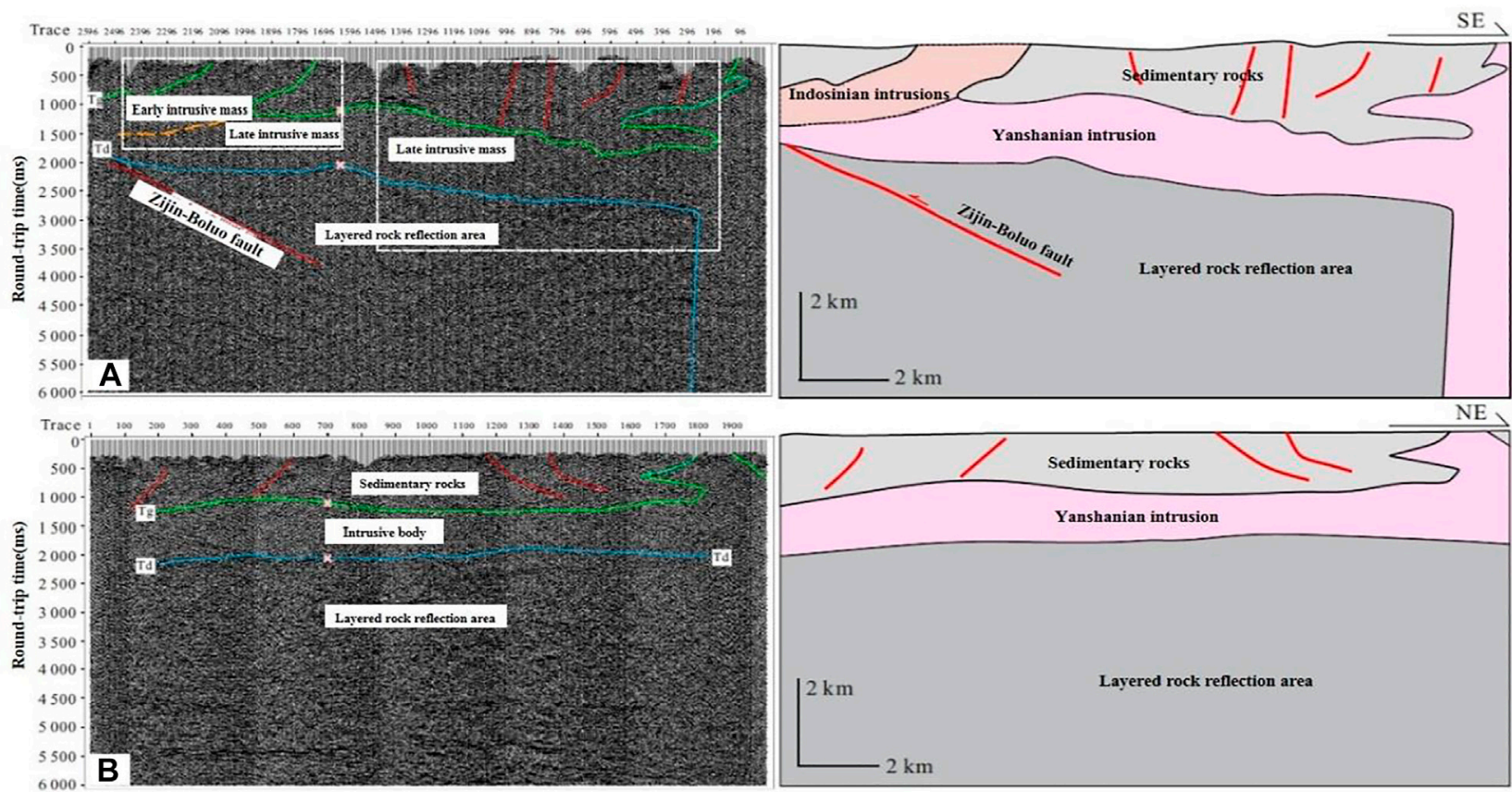

FIGURE 10 | Profiles of seismic inversion, (A), (B) and its geological interpretation in Huangshadong area (Kuang et al., 2020). 
$(\mathrm{Y}+\mathrm{Nb})-\mathrm{Rb}$ diagram (Figures 9C,D), samples are plotted within the scope of the volcanic island arc granite (VAG), within plate granite (WPG) and sys-collision granite (syn-COLG). Combined with the trace element characteristics of the samples, it can be inferred that the samples of this period is similar to the granite formed in $\sim 135 \mathrm{Ma}$. Li Jianhua (2013) divided the acidic magmatic rocks of the Cretaceous into three periods: Adakite and gneis-like granite at 145-137 Ma, calc-alkaline granite at 136$118 \mathrm{Ma}$ and related volcanic rocks, bimodal volcanic rocks at 107-90 Ma and A-type granite at 145-137 Ma. This is similar to what we found in this study.

\section{Discussion on the Burial Depth of Deep Granite \\ Seismic Profiles in Huangshadong Area of Guangdong Province}

The two seismic profiles in Huangshadong area of Guangdong Province (Figures 10A,B) (Kuang et al., 2020) reflect the sedimentary strata overlying granites. The sedimentary strata in the study area have undergone complex tectonic evolution, repeated tectonic uplifting, compression folding and fault processes have caused the sedimentary strata to have dramatic occurrence changes in a small range, and multiple periods of magmatic intrusion and eruption have complicated the contact relationship strata and rock mass. Therefore, the spatial continuity of stratigraphic interface and the stability of fault characteristics in seismic profile are poor in different ages.

According to the profiles of seismic inversion (Figure 10), the buried depth of $\mathrm{Tg}$ (interface between sedimentary strata and granites) is generally located between 1,000-1,900 ms (corresponding depth of 1,400-2,200 m), and the seismic phase characteristics of $\mathrm{Tg}$ interface are significantly different from each other. The granite core sample from Well Huire one is similar to Yanshanian granite, indicating that seismic facies unit may correspond to the intrusive body in Yanshanian period, emplaced from the deep crust upward into shallow rock bed. 2050-2,900 ms (corresponding to the depth of 3,600-4,800 m) for the bedrock under the bottom of the interface $(\mathrm{Td})$, reflection of geological units in phase axis continuity better, different from the adjacent granite rock mass. It may be older sedimentary rocks or metamorphic rocks with certain stratification.

\section{Apparent Resistivity Inversion Profiles in North Hainan Province}

In the area outside the east of Fushan Depression of Hainan Province, wide field electromagnetic detection has been conducted (Figure 11) (Tan et al., 2020). From the resistivity
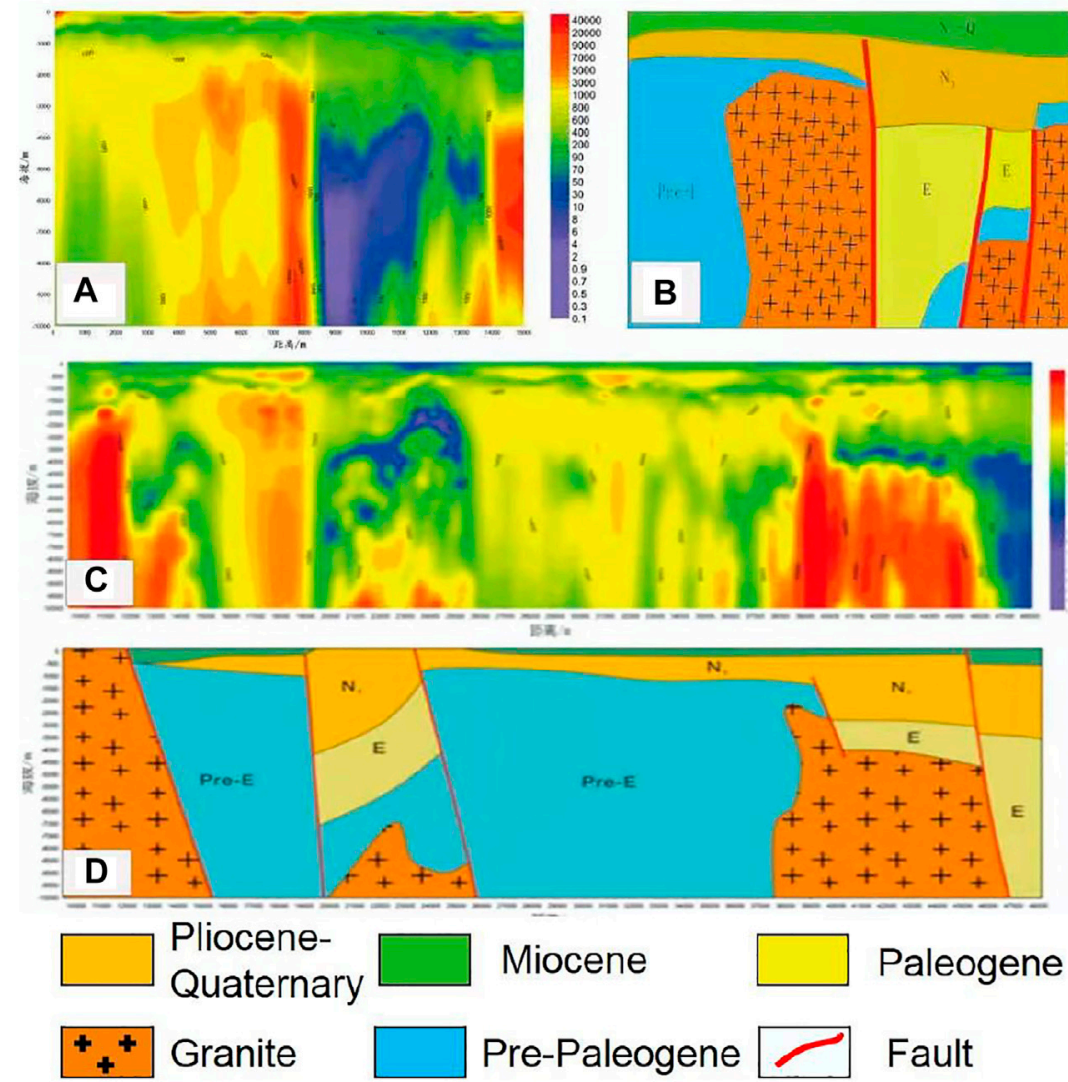

FIGURE 11 | Profiles of apparent resistivity inversion based on wide field electromagnetic method and corresponding geological interpretation in North Hainan Province (Tan et al., 2020). 
profile of the final inversion processing, a set of high resistance developed in the west of the L1 line and a set of low resistance developed in the east (Figure 11A). The two parts were clearly bounded and featured with great difference. Based on regional geological analysis, the corresponding geological model is established (Figure 11B), and it is preliminarily considered that the high resistivity in the east is a set of intrusive concealed granite, and the low resistivity in the west is a paleoproximal sedimentary stratum, and the middle fault controls its scale. According to the analysis of geological model, the basement of North Hainan area is composed of pre-Mesozoic sedimentary rocks and granite intrusive bodies, and the rock strains develop. The fault-block-graben-barrier is developed in this area, and the structure is complex. The faultblock-graben-barrier structure is developed in the east, which is similar to Fushan Depression. Moreover, the distribution of Paleogene strata is obviously controlled by the size of the fault depression. The L4 line extends southward to the granite outflowing area. According to the resistivity result diagram (Figure 11C), the electrical characteristics of the outflowing granite are similar to those of the latent granite (Figure 11D). By comparing the two survey lines, the latent high-resistivity stability exists. In the intersection area where survey line L1 and survey line L4 meet, the granite has a large scale, a buried depth of about $3000 \mathrm{~m}$, and an overburden, which is a potential favorable area for deep high-temperature geothermal exploration.

\section{The Significance of Granite to the Geothermal Energy}

The heat source of geothermal energy mainly include heat transfer in the mantle, local anomalous heat transfers in the crust (magma chamber), and radioactive heat generation from granitic rocks. Surface heat flow, as a direct reflection of deep heat, usually accounts for $40-60 \%$ of the heat flow from the mantle. The heat flow caused by radioactive heat generation in the crust is slightly less than that from the mantle, accounting for about $40 \%$ and up to $50 \%$ in some areas (Wang Shejiao et al., 1999). The terrestrial heat flow value in the study area is about $70 \mathrm{~mW} / \mathrm{m}^{2}$, which is higher than the average terrestrial heat flow value of $64.2 \mathrm{~mW} / \mathrm{m}^{2}$ in South China (Yuan et al., 2006). The Yanshannian granite in the Southeast Coastal region has a high thermal conductivity, with an average thermal conductivity of $3.15 \mathrm{~W} / \mathrm{mK}$. This means that the granite in the deep part with a thickness of up to $3.5 \mathrm{~km}$ can better conduct the heat in the deeper part to the near surface. Consequently, the mantle heat gets better performance on the surface, which promotes the formation of the geothermal energy in the area.

Former research suggested that the heat generated by radioactive element decay in rocks is one of the main sources of Earth heat (Huang Shaopeng, 1992), and it is also a major factor controlling the temperature field distribution in the lithosphere (Sclater et al., 1980). Previous scholars proposed that the lower crust has little influence on the surface heat flow value (Hasterok and Chapman, 2007), and the research on the lower crust inclusion also shows that the radioactive heat generation rate of rocks in the lower crust is very low (Rudnick and Fountain, 1995). Thus, the heat source in the area mainly comes from the decay of radioactive elements in the middle and upper crust, and depends on the abundance of thermogenic elements, such as ${ }^{238} \mathrm{U},{ }^{232} \mathrm{Th}$, and ${ }^{40} \mathrm{~K}$ (Rybach and Buntebarth, 1984).

Previous studies have analyzed the overall heat generation rate of radioactive elements in the study area shown as low heat generation rate of strata and high heat generation rate of granitic rocks (Kuang et al., 2020). The value of heat generation rate from Yanshanian rocks is far higher than the average global continental upper crust rock, with the average value of $5.50 \mu \mathrm{W} / \mathrm{m}^{3}$ and the maximum value up to $9.15 \mu \mathrm{W} / \mathrm{m}^{3}$. It is also much higher than the average measured value of the radioactive heat generation rate of granitic rocks in other regions, which was about $2.5 \mu \mathrm{W} / \mathrm{m}^{3}$ (Rybach, 1988), 3.3-3.8 $\mu \mathrm{W} / \mathrm{m}^{3}$ (Wollenberg and Smith, 1987) and $3.0 \mu \mathrm{W} / \mathrm{m}^{3}$ (Rybach and Buntebarth, 1984).

This is mainly attributed to Yanshanian granites formed from remelting of pre-Cambian rocks in this region (Xu et al., 2007), The rocks have been experienced the redistribution process, and enriched large ion lithophile elements (LILE) of rocks, lead to extreme enrichment of LILE (including Th, U, K element), and then forming high value of heat generation rate in granites. Consequently, the high thermal conductivity and high radioactive thermogenic elements of Yanshanian granites are the reasons for the formation of high-temperature geothermal in this area.

\section{The Significance of Granite to Deep Geothermal in Southeast China}

Large-scale granite events occurred in the coastal area of southeast China during Mesozoic, especially Yanshannian (Li, et al., 2014; Gao et al., 2014; Deng et al., 2016). The Yanshannian granitoids magmatism in the southeast coastal area has a general trend from west to east, and the emplacement age of the rocks from old to young. Due to the high radioactive heat generation rate of granitoids, granitoids provide a direct heat source for crustal heat generation. Therefore, the widespread occurrence of granitoids magmatic activity in Mesozoic provides a significant condition for the formation of geothermal resources. The terrestrial heat flow value is one of the important geothermal geological indexes for the occurrence and distribution of geothermal resources. The terrestrial heat flow value in the southeast coastal area of China is obviously affected by the tectonic background (Hu and Wang, 1994; Zhang et al., 2020): The overall tectonic activity in the northwestern basin is relatively stable, showing a lower terrestrial heat flow value, while the southeastern coast shows a higher terrestrial heat flow value. As the overall heat flow value gradually increases from west to east, the local deep thermal structure controls the ground heat flow value. As shown in Figure 2, Fujian and YangjiangMaoming reached the highest ground heat flow value, reaching more than $95 \mu \mathrm{W} / \mathrm{m}^{3}$. Radiogenic heat is one of the main sources of heat in the lithosphere. $\mathrm{U}$, Th and natural radio isotope ${ }^{40} \mathrm{~K}$ are the main thermal elements. According to the Zhao Ping et al. (1995), most of the southeast coastal areas are in the range of high heat generation rate, basically higher than $2.1 \mu \mathrm{W} / \mathrm{m}^{3}$. Especially in the whole province of Guangdong, 
southern Jiangxi and southern Fujian, the large area of granite outcrops, the heat generation rate background is more than $2.8 \mu \mathrm{W} / \mathrm{m}^{3}$, such a large area of high heat generation rate area is rare. Radioactive heat generation is the main source of crustal heat generation, which is of great significance to the occurrence of dry hot rock resources. The southeast coastal area, especially Huizhou and Sanhui of Guangdong Province, has a good prospect of exploration and development of geothermal resources.

Based on produced heat rate of typical magmatic rocks in Guangdong, Jiangxi and southeast of Fujian, previous scholars indicated that the radioactive heat production rate of most granitic rocks is greater than the average heat production rate of the Earth's crust, signifying that the Yanshanian granite radioactive heat production have great contribution to regional heat (Zhao et al., 1995). Meanwhile, the southeastern coastal area has basically the same tectonic evolution pattern in Mesozoic (Wang et al., 2013), which is controlled by Paleo-Pacific plate subduction during the Yanshanian period and experienced largescale magmatic events.

Based on seismic inversion in the area, it is clear that in this area and the surrounding granite areas, based on $\mathrm{P}$ wave inversion of deep seismic profiles, shows $5-10 \mathrm{~km}$ depth of granite. A reasonable speculation in the area is that Yashaninan granites have great potential for the geothermal reservoirs. Moreover, the Moho surface in the whole southeast region is relatively shallow and the temperature of the Moho surface is relatively high (Zhang et al., 2018). The deep granite can promote the heat transfer of the mantle to the surface. In summary, Yanshaninan granite in the southeast coastal area is of great significance to the geothermal energy system.

\section{CONCLUSION}

1) The age of granite reservoirs are mainly 94.7-138.4 Ma, mainly late Yanshanian period. The geochemical characteristics of rocks show they are formed in the

\section{REFERENCES}

Bertani, R. (2012). Geothermal Power Generation in the World 2005-2010 Update Report. Geothermics 41, 1-29. doi:10.1016/j.geothermics.2011.10.001

Bertani, R. (2016). Geothermal Power Generation in the World 2010-2014 Update Report. Geothermics 60, 31-43. doi:10.1016/ j.geothermics.2015.11.003

Brown, D. W., Duchane, D. V., Heiken, G., and Hriscu, V. (2012). Mining the Earth's Heat: Hot Dry Rock Geothermal Energy. Heidelberg: Springer Science \& Business Media. doi:10.1007/978-3-540-68910-2

Deng, J., Feng, Y., Di, Y., Cui, L., Xiao, Q., and Guo, S. S. (2016). The Instrusive Spatial Temporal Evolutional Framework in the Southeast China. Geol. Rev. 62 (1), 3-16.

Deng, Y. F., Li, J. T., and Peng, T. P. (2019). Lithospheric Structure in the Cathaysia Block (South China) and its Implication for the Late Mesozoic Magmatism. Physics of the Earth and Planetary Interiors, 24-34.

Frost, B. R., Barnes, C. G., Collins, W. J., Arculus, R. J., Ellis, D. J., and Frost, C. D. (2001). A Geochemical Classification for Granitic Rocks. J. Pet. 42 (11), 2033-2048. doi:10.1093/petrology/42.11.2033 subduction zone signature, indicating they are formed related to the extensional environment formed by retractable subduction of the ancient Pacific plate.

2) Deep Yanshanian granites promote mantle heat transfer to the surface and increase the proportion of mantle heat flow in the heat flow value. Yanshanian granite has high content of Th, U, and $\mathrm{K}$, and its radioactive decay heat generation provides heat source for the high-temperature geothermal in the study area. The combined action of the two forms the high-temperature geothermal energy in the study area.

3) The deep granite in the study area has huge potential for geothermal resources. The geothermal genesis model in the study area has reference significance for the understanding of geothermal in the southeast region or the calculation of geothermal reserves.

\section{DATA AVAILABILITY STATEMENT}

The original contributions presented in the study are included in the article/supplementary material, further inquiries can be directed to the corresponding author.

\section{AUTHOR CONTRIBUTIONS}

$\mathrm{JL}$ is responsible for experimental design, $\mathrm{HZ}$ and $\mathrm{YZ}$ are responsible for providing overall ideas, JF is responsible for data analysis, $\mathrm{YZ}$ and $\mathrm{MW}$ is responsible for instrument operation.

\section{FUNDING}

This study was financially supported by National Key Research and Development Project (No. 2019YFC0604903), National Natural Science Foundation of China (Grant No. U20B6001) and consultation research project of Chinese Academy of Engineering (No. 2019-XZ-35-04 and No. 2021-XZ-16-01).

Gao, W., Wang, Z., Li, C., and Wang, D. (2014). Zircon U-Pb Geochronological, Geochemistry and Tectonic Implication of Lndosinian Granite from southeastern Zhejiang, South China. Acta Geologica Sinica 88 (6), 1055 1067.

Genter, A., Traineau, H., Dezayes, C., Elsass, P., and Villemin, T. (1995). Fracture Analysis and Reservoir Characterization of the Granitic Basement in the HDR Soultz Project (France). Geothermal Sci. Tech. 4 (3), 189-214.

Goldstein, B. A., Hill, A. J., and Long, A. (2008). Hot Rocks in Australia National Overview. ASEG Extended Abstr. 1, 1.

Gong, J., and John Chen, Y. (2014). Evidence of Lateral Asthenosphere Flow beneath the South China Craton Driven by Both Pacific Plate Subduction and the India-Eurasia continental Collisin. Terra Nova 26 (1), 55-63. doi:10.1111/ ter.12069

Hasterok, D., and Chapman, D. S. (2007). Continental Thermal Isostasy: 2. Application to North America. J. Geophys. Res. Solid Earth 112 (B6). doi:10.1029/2006jb004664

Hu, S., He, L., and Wang, J. (2000). Heat Flow in the Continental Area of China: A New Data Set. Earth Planet. Sci. Lett. 179 (2), 407-419. doi:10.1016/s0012$821 x(00) 00126-6$

$\mathrm{Hu}$, S., and Wang, J. (1994). Heat Flow Characteristics of Orogenic Belts in southeastern China. Geol. Rev. 40 (5), 387-394. 
Huang, S. P. (1992). Variations of Heat Flow and Crustal Thickness in the Continental Area of China. Chin. J. Geophys. 35 (4), 441-450.

Kelemen, P. B., Hanghøj, K., and Greene, A. R. (2003). One View of the Geochemistry of Subduction - Related Magmatic Arcs, with an Emphasis on Primitive Andesite and Lower Crust. Treatise Geochem. 3, 659.

Kuang, J., Qi, S., and Wang, S. (2020). Granite Intrusion in Huizhou, Guangdong Province and its Geothermal Implications. Earth Sci. 45 (2020), 1466-1480.

Li, D. W., and Wang, Y. X. (2015). Major Issues of Research and Development of Hot Dry Rock Geothermal Energy. Earth Sci. 40 (11), 1858-1869.

Li, J., Zhang, Y., Dong, S., and Johnston, S. T. (2014). Cretaceous Tectonic Evolution of South China: a Preliminary Synthesis. Earth-Science Rev. 134, 98-136. doi:10.1016/j.earscirev.2014.03.008

Li, L., Luo, J., Chen, Q., Shi, D., Yun, J., and Li, T. (2019). Discovery of Silurian schist from Well J6 in Chagan Sag, Yin'e Basin: Evidence from Zircon U-Pb Geochronology. Geology in China 46 (1), 209-201

Li, Sanzhong., Zang, Yibo., and Wang, P. (2017). Earth Sci. Front. 24 (4), 213-225.

Li, X. H., Li, Z. X., Li, W., Ying, L., Yuan, C., Wei, G., et al. (2007). U- Pb Zircon, Geochemical and Sr-Nd-Hf Isotopic Constraints on Age and Origin of Jurassic I- and A-Type Granites from Central Guangdong, SE China: A Major Igneous Event in Response to Foundering of a Subducted Flat-Slab? Lithos 96 (1-2), 186-204. doi:10.1016/j.lithos.2006.09.018

Li, Z.-X., and Li, X.-H. (2007). Formation of the $1300-\mathrm{km}$-wide Intracontinental Orogen and Postorogenic Magmatic Province in Mesozoic South China: A FlatSlab Subduction Model. Geol. 35 (2), 179. doi:10.1130/g23193a.1

Liu, K., Li, Z., Xu, W., Ye, H., Zhao, X., Hu, Y., et al. (2016). Geochemical Characteristics and Metallogenic Regularity of Mesozoic Magmatic Rocks in South China [J]. J. Mineralogy, Pet. Geochem. 35 (6), 1141-1155.

Liu, Y. S., Hu, Z. C., Gao, S., Günther, D., Xu, J., Gao, C. G., et al. (2008). In Situ analysis of Major and Trace Elements of Anhydrous Minerals by LA-ICP-MS without Applying an Internal Standard. Chem. Geology 257 (1-2), 34-43. doi:10.1016/j.chemgeo.2008.08.004

Ludwig, K. R. (2003). Isoplot 3.00: A Geochronological Toolkit for Microsoft Excel. Berkeley: Berkeley Geochronology Center Special Publication, 4-70.

Lund, J. W., and Boyd, T. L. (2016). Direct Utilization of Geothermal Energy 2015 Worldwide Review. Geothermics 60, 66-93. doi:10.1016/ j.geothermics.2015.11.004

Pan, Z., Zhang, Q., Chen, G., Jiao, S., Du, X., Miao, X., et al. (2017). The Relationship between the Mesozoic Magmatic Activity and Plate Subduction in Eastern China: The Relation between the Zhe-Fujian and the Japanese Arc and the Andean Arc and its Significance [J]. Acta Petrologica Sinica 33 (5), $1507-1523$.

Pearce, J. A., Harris, N. B. W., and Tindle, A. G. (1984). Trace Element Discrimination Diagrams for the Tectonic Interpretation of Granitic Rocks. J. Pet. 25 (4), 956-983. doi:10.1093/petrology/25.4.956

Richards, H. G., Savage, D., and Andrews, J. N. (1992). Granite-water Reactions in an Experimental Hot Dry Rock Geothermal Reservoir, Rosemanowes Test Site, Cornwall, U.K. Appl. Geochem. 7 (3), 193-222. doi:10.1016/0883-2927(92) 90038-5

Rudnick, R. L., and Fountain, D. M. (1995). Nature and Composition of the Continental Crust: A Lower Crustal Perspective. Rev. Geophys. 33 (3), 267. doi:10.1029/95rg01302

Rybach, L., and Buntebarth, G. (1984). The Variation of Heat Generation, Density and Seismic Velocity with Rock Type in the Continental Lithosphere. Tectonophysics 103 (1-4), 335-344. doi:10.1016/0040-1951(84)90095-7

Rybach, L., 1988. Determination of Heat Production Rate. Handbook of Terrestrial Heat Flow Density Determinations.

Sclater, J. G., Jaupart, C., and Galson, D. (1980). The Heat Flow through Oceanic and Continental Crust and the Heat Loss of the Earth. Rev. Geophys. 18 (1), 269-311. doi:10.1029/rg018i001p00269
Sun, S.-s., and McDonough, W. F. (1989). Chemical and Isotopic Systematics of Oceanic Basalts: Implications for Mantle Composition and Processes. Geol. Soc. Lond. Spec. Publications 42 (1), 313-345. doi:10.1144/ gsl.sp.1989.042.01.19

Tan, H., Lin, F., Wen, J., Li, J., and Fan, Y. (2020). Application of Wide Field Electromagnetic Method in the Exploration of Deep Dry Hot Rocks in Hainan Province. China Pet. Chem. Stand. Qual. (4), 150-152.

Wang, D., and Shen, W. (2003). Granite Genesis and Crustal Evolution in southeastern China. Earth Sci. Front. 10 (3), 209-220.

Wang, S. J., Hu, S. B., and Wang, S. J. (1999). The Geothermal Effect of Radioactive Heat Generation and its Signif-icance to Hydrocarbon Maturation in Tarim Basin. Pet. Exploration Dev. 26 (5), 36-38.

Wang, Y., Fan, W., Zhang, G., and Zhang, Y. (2013). Phanerozoic Tectonics of the South China Block: Key Observations and Controversies. Gondwana Res. 23 (4), 1273-1305. doi:10.1016/j.gr.2012.02.019

Wollenberg, H. A., and Smith, A. R. (1987). Radiogenic Heat Production of Crustal Rocks: An Assessment Based on Geochemical Data. Geophys. Res. Lett. 14 (3), 295-298. doi:10.1029/gl014i003p00295

Wu, F., Li, X., Yang, J., and Zheng, Y. (2007). Discussions on the Petrogenesis of Granites [J]. Acta Petrologica Sinica 23 (6), 1217-1238.

Xi, Y., Wang, G., Liu, S., Zhao, Y., and Hu, X. (2018). The Formation of a Geothermal Anomaly and Extensional Structures in Guangdong, China: Evidence from Gravity Analyses. Geothermics 72, 225-231. doi:10.1016/ j.geothermics.2017.11.009

Xu, X. S., O'Reilly, S. Y., Griffin, W. L., Wang, X., Pearson, N., and He, Z. (2007). The Crust of Cathaysia: Age, Assembly and Reworking of Two Terranes. Precambrian Res. 158 (1-2), 51-78. doi:10.1016/j.precamres.2007.04.010

Yuan, Y. S., Ma, Y. S., Hu, S. B., Guo, T., and Fu, X. (2006). Present - Day Geothermal Characteristics in South China. Chin. J. Geophys. 49 (4), 1118-1126. doi:10.1002/cjg2.922

Zhang, J., Wang, B. Y., Tang, X. C., and Dong, M. (2018). Temperature Structure and Dynamic Background of Crust and Mantle beneath the High Heat Flow Area of the South China Continental Margin. Chin. J. Geophys. 61 (10), 3917-3932.

Zhang, Y., Luo, J., and Feng, J. (2020). Characteristics of Geothermal Reservoirs and Utilization of Geothermal Resources in the Southeastern Coastal Areas of China. Journal of Groundwater Science and Engineering 8 (2), 134-142.

Zhao, P., Wang, J., and Wang, J. A. (1995). Characteristics of Heat Production Distribution in SE China. Acta Petrologica Sinica 11 (3), 292-305.

Zhou, X., Sun, T., Shen, W., Shu, L., and Niu, Y. (2006). Petrogenesis of Mesozoic Granitoids and Volcanic Rocks in South China: A Response to Tectonic Evolution. Episodes 29 (1), 26-33. doi:10.18814/epiiugs/2006/v29i1/004

Conflict of Interest: The authors declare that the research was conducted in the absence of any commercial or financial relationships that could be construed as a potential conflict of interest.

Publisher's Note: All claims expressed in this article are solely those of the authors and do not necessarily represent those of their affiliated organizations, or those of the publisher, the editors and the reviewers. Any product that may be evaluated in this article, or claim that may be made by its manufacturer, is not guaranteed or endorsed by the publisher.

Copyright $\odot 2021$ Zheng, Luo, Zhang, Feng, Zeng and Wang. This is an open-access article distributed under the terms of the Creative Commons Attribution License (CC $B Y$ ). The use, distribution or reproduction in other forums is permitted, provided the original author(s) and the copyright owner(s) are credited and that the original publication in this journal is cited, in accordance with accepted academic practice. No use, distribution or reproduction is permitted which does not comply with these terms. 